\title{
Functional Renormalization Group Approach for Inhomogeneous One-Dimensional Fermi Systems with Finite-Ranged Interactions
}

\author{
Lukas Weidinger, ${ }^{1}$ Florian Bauer ${ }^{1}$ and Jan von Delft ${ }^{1}$ \\ ${ }^{1}$ Arnold Sommerfeld Center for Theoretical Physics and Center for NanoScience, \\ Ludwig-Maximilians-Universität München, Theresienstrasse 37, D-80333 München, Germany
}

(Dated: September 14, 2021)

\begin{abstract}
We introduce an equilibrium formulation of the functional renormalization group (fRG) for inhomogeneous systems capable of dealing with spatially finite-ranged interactions. In the general third order truncated form of fRG, the dependence of the two-particle vertex is described by $\mathcal{O}\left(N^{4}\right)$ independent variables, where $N$ is the dimension of the single-particle system. In a previous paper [Bauer et al., Phys. Rev. B 89, 045128 (2014)], the so-called coupled-ladder approximation (CLA) was introduced and shown to admit a consistent treatment for models with a purely onsite interaction, reducing the vertex to $\mathcal{O}\left(N^{2}\right)$ independent variables. In this work, we introduce an extended version of this scheme, called the extended coupled ladder approximation (eCLA), which includes a spatially extended feedback between the individual channels, measured by a feedback length $L$, using $\mathcal{O}\left(N^{2} L^{2}\right)$ independent variables for the vertex. We apply the eCLA in a static approximation and at zero temperature to three types of one-dimensional model systems, focussing on obtaining the linear response conductance: First, we study a model of a quantum point contact (QPC) with a parabolic barrier top and onsite interactions. In our setup, where the characteristic length $l_{x}$ of the QPC ranges between approximately 4-10 sites, eCLA achieves convergence once $L$ becomes comparable to $l_{x}$. It also turns out that the additional feedback stabilizes the fRG-flow. This enables us, second, to study the geometric crossover between a QPC and a quantum dot, again for a one-dimensional model with onsite interactions. Third, the enlarged feedback also enables the treatment of a finite-ranged interaction extending over up to $L$ sites. Using a simple estimate for the form of such a finite-ranged interaction in a QPC with a parabolic barrier top, we study its effects on the conductance and the density. We find that for low densities and sufficiently large interaction ranges the conductance develops additional features, and the corresponding density shows some fluctuations that can be interpreted as Friedel oscillations arising from a renormalized barrier shape with a rather flat top and steep flanks.
\end{abstract}

\section{INTRODUCTION}

The functional renormalization group (fRG) is a wellestablished tool for studying interacting many-body systems [1-6]. This technique treats interactions using an RG-enhanced perturbation theory and is known to provide an efficient way to treat correlations. In particular, fRG can be used to treat spatially inhomogeneous systems, represented by a discretized model with $N$ sites. For example, about $N \sim 10^{2}$ sites are required to represent the electrostatic potential of a quasi-one-dimensional point contact in a manner that is sufficiently smooth to avoid finite-sitze effects [7]. The corresponding twoparticle vertex has $\mathcal{O}\left(N^{4}\right) \sim 10^{8}$ independent spatial components. To make numerical computations feasible, simplifying approximations have to be made to reduce the number of components used to describe the vertex. Such a scheme, called the coupled-ladder approximation (CLA), was proposed in Ref. 7 for the case of onsite interactions. Bauer, Heyder and von Delft (BHD) [8] supplied a detailed description of the CLA which is in principle applicable to systems of arbitrary dimensionality. The CLA is implemented within the context of generic, third-ordertruncated fRG, meaning that all vertices with three and higher particle number are set to zero throughout the whole flow. In this paper we generalize this scheme to be able to treat finite-ranged interactions. Since the central aim of our scheme is to extend the spatial range over which information is fed back into the RG flow, we call our scheme the extended coupled-ladder approxima- tion (eCLA).

The basic idea of the CLA, and by extension the eCLA, lies in reducing the number independent components of the vertex by decomposing it into several interaction channels and then establishing a consistent approximation by controlling the amount of feedback between the individual channels. This strategy follows that used in Refs. 4 and 9 in the context of the single-impurity Anderson model. For a model with short-ranged interactions, this approach reduces the number of independent quantities in the vertex to order $\sim \mathcal{O}\left(N^{2}\right)$. From a perturbative point of view, this treatment is exact in second order in the interaction and amounts to summing up approximate contributions from a large class of diagrams, including mutual feedback between the different interaction channels. The eCLA generalizes the CLA by extending spatial feedback between the channels. As a control parameter for this extended feedback we introduce a feedback length $L$, where $L=0$ corresponds to the previous approximation scheme used by BHD, while $L=N-1$ includes the full fRG flow in second order. $L$ thus serves as a control parameter for the number of independent spatial components of the vertex, which scales as $\sim \mathcal{O}\left(N^{2} L^{2}\right)$. Moreover, the longer-ranged feedback allows us also to treat interactions with finite range up to $L_{U}$ sites (with $L_{U} \leq L$ ) in a manner that is exact to second order in the interaction.

In this paper, we present a detailed account of the eCLA, and apply it to two one-dimensional (1D) fermionic systems, modeled to describe the lowest 1D 
subband of a quantum point contact (QPC) or a quantum dot (QD), respectively. We develop the eCLA for systems described by a Hamiltonian of the form

$$
\hat{H}=\sum_{i j, \sigma} h_{i j}^{\sigma} d_{i \sigma}^{\dagger} d_{j \sigma}+\frac{1}{2} \sum_{i j, \sigma \sigma^{\prime}} U_{i j} \hat{n}_{i \sigma} \hat{n}_{j \sigma^{\prime}}\left(1-\delta_{i j} \delta_{\sigma \sigma^{\prime}}\right),
$$

where $h^{\sigma}$ and $U$ are real, symmetric matrices, $d_{j \sigma}^{\dagger}$ creates an electron in single particle state $j$ with spin $\sigma(=\uparrow, \downarrow$ or $+,-$, with $\bar{\sigma}=-\sigma)$, and $n_{j \sigma}=d_{j \sigma}^{\dagger} d_{j \sigma}$. In the context of the applications presented here, we refer to the quantum number $j$ as the "site index". Our eCLA scheme requires the interaction to have a finite range $L_{U} \leq L$, such that

$$
U_{i j}=0 \quad \text { if }|i-j|>L_{U} .
$$

Models of this form, but with onsite interactions $\left(U_{i j}=\right.$ $\left.U \delta_{i j}\right)$, have been used to study both QPC and QD systems [7]. To describe a QPC, $h_{i j}^{\sigma}$ is taken to represent a one-dimensional tight-binding chain, with a potential barrier with parabolic top, whereas for a QD it is chosen to represent a double-barrier potential. The noninteracting physics of both models is well known, whereas the effect of interactions, especially for the QPC, are still a topic of ongoing discussions [10-12]. For the QPC the conductance is quantized [13-15] in units of the conductance quantum $G_{Q}=2 e^{2} / h$, but shows an additional shoulder at approximately $0.7 G_{Q}$. This regime, in which other observables show anomalous behavior too [16-18], is commonly known as the "0.7-anomaly". The latter has been studied in [7] using a model of the above form, with purely onsite interactions. However, to examine the effect of gate-induced screening in a QPC, one needs to consider finite-ranged interactions. This goal serves as the main motivation for developing the eCLA put forth in this paper.

We remark that the QD and QPC models considered here provide a meaningful testing ground for the eCLA, since lowest-order perturbation theory would not yield an adequate treatment of the correlation effects expected to occur: the Kondo effect for QDs and the 0.7-anomaly for QPCs. Although some aspects of the latter can be understood in terms of a simple Hartree picture [7], the interaction strength needed to yield phenomenological behavior typical of the 0.7 -anomaly is sufficiently large that lowest-order perturbation theory is inadequate.

The numerical results presented here were all obtained using the eCLA in a static approximation, which neglects the frequency-dependence of the two-particle vertex (after which the approach no longer is exact to second order). Nevertheless, BHD have shown that for a QPC model with onsite interactions, the CLA with a static approximation leads to reasonable results for the conductance step shape, though it does produce some artifacts regarding the pinch-off gate voltage when the interaction strength is increased. We find the same to be true for the static eCLA, with the artifacts becoming more pronounced with increasing interaction range, but the step shape behaving in a physically reasonable manner.
We use the eCLA for three studies of increasing complexity. (i) We present static eCLA results for a QPC model with short-ranged interaction and successively increase the feedback length $L$. This systematically improves the treatment of RG-feedback between the various fRG channels, and for sufficiently large $L$ converges to the full solution of the generic, third-order-truncated static fRG. For the models we consider here, where the characteristic length $l_{x}$ of the parabolic QPC potential barrier varies between approximately $4-10$ sites, we find that convergence in $L$ is achieved once $L$ becomes comparable to $l_{x}$. For such systems, the eCLA scheme thus speeds up the calculation relative to the full generic, third-order-truncated static fRG by a factor of $10^{3}$, without any loss of accuracy. (ii) Furthermore, it turns out that the eCLA's enhanced feedback leads to a more stable fRG flow than the CLA scheme, since each interaction channel acts more strongly to limit the tendencies other channels might have to diverge during the fRG flow. This enables us to study the geometric crossover between a QPC and a QD where the barrier top stays close to the chemical potential. This setup features a high local density of states (LDOS) at the chemical potential, and as a result turns out to be intractable when using the CLA without enhanced feedback [19]. In contrast, the eCLA is able to treat this challenging crossover very nicely. (iii) Finally, we illustrate the potential of the eCLA to deal with finite-ranged interactions in a setting where the physics of screening comes into play, namely a QPC model with an interaction whose range extends over up to $N$ sites. The purpose of this study is mainly methodological, i.e. we do not aim here to achieve a fully realistic treatment of screening in a QPC. Nevertheless, the results are interesting: for a sufficiently long ranged interaction and sufficiently low density, there exists a parameter regime where we find additional features in the conductance and corresponding $2 k_{F}$ density fluctuations within the QPC.

The paper has three main parts. The first part (section II) develops our improved eCLA feedback scheme. The second part (section III) studies its consequences for QPC and QD models with onsite interaction, focusing on the effects of increasing the feedback length $L$. Finally, the third part (section IV) is devoted to finiteranged interactions. We estimate the approximate form and strength of the interaction to be used for a $1 \mathrm{D}$ depiction of a QPC and show some preliminary results for the conductance and density profile of such a system depending on the screening properties. A detailed study of the physics of long-ranged interactions in QPCs is beyond the scope of this work and left as a topic of future investigation.

\section{FRG FLOW EQUATIONS}

Before we introduce our new eCLA scheme, we give a short overview over the general idea and the usual approximations made in fRG. Since numerous detailed treatments of fRG are available, and since our work 
builds on that of BHD, the discussion below is very brief and structured similarly to that in Ref. 8. The basic idea of fRG is to introduce a flow parameter $\Lambda$ in the bare propagator of the theory in such a way that for $\Lambda=\Lambda_{i}=\infty$, the structure of the resulting vertex functions are very simple. With our choice for $\Lambda$ (described later) all but the two-particle vertex will vanish,

$$
\gamma_{2}^{\Lambda_{i}}=v \quad \gamma_{n}^{\Lambda_{i}}=0 \quad(n \neq 2),
$$

where $v$ is the bare vertex. For the final value of the flow parameter $\Lambda=\Lambda_{f}=0$, one recovers the full bare propagator and hence the full theory:

$$
\mathcal{G}_{0}^{\Lambda} \rightarrow \mathcal{G}_{0}, \quad \text { with } \quad \mathcal{G}_{0}^{\Lambda_{i}}=0, \quad \mathcal{G}_{0}^{\Lambda_{f}}=\mathcal{G}_{0} .
$$

The RG flow is described by a hierarchy of coupled differential equations for the one particle irreducible (1PI) $n$-particle vertex functions $\gamma_{n}$,

$$
\frac{d}{d \Lambda} \gamma_{n}^{\Lambda}=\mathcal{F}\left(\Lambda, \mathcal{G}_{0}^{\Lambda}, \gamma_{1}^{\Lambda}, \ldots, \gamma_{n+1}^{\Lambda}\right)
$$

Integrating this system from $\Lambda=\Lambda_{i}$ to $\Lambda=0$ yields in principle a full description of all interaction vertices. In practice, one can of course not treat an infinite hierarchy of flow equations and has to truncate it at some point. In our form of third-order truncated fRG, we incorporate the one- and two-particle vertex into the flow, but set all vertices with three or more particles to zero

$$
\frac{d}{d \Lambda} \gamma_{n}=0 \quad(n \geq 3) .
$$

We thus retain only the flow of the self-energy, $\Sigma=-\gamma_{1}$, and the flow of the two-particle vertex $\gamma_{2}$. This differential equation can then be solved numerically, using a standard Runge-Kutta method. As we will see shortly, the flow of the vertex consists of three different parquet-like channels which are coupled to the flow of the self-energy and also directly to each other. This simultaneous treatment moderates competing instabilities in an unbiased way.

In principle, the form of the fRG flow equations depends on the choice of the flow parameter, even if in most cases they take the form stated below. In our work, we choose the $\Lambda$-dependence of the bare propagator to take the form of an infrared cutoff

$$
\mathcal{G}_{0}^{\Lambda}\left(\omega_{n}\right)=\Theta_{T}\left(\left|\omega_{n}\right|-\Lambda\right) \mathcal{G}_{0}\left(\omega_{n}\right), \Lambda_{i}=\infty, \Lambda_{f}=0 .
$$

We use the Matsubara formalism with the frequencies $\omega_{n}$ defined to be purely imaginary,

$$
\omega_{n}=i T \pi(2 n+1),
$$

and $\Theta_{T}$ is a step function broadened on the scale of temperature.

Using this cutoff, one can derive the fRG equations in the standard way, see e.g. Refs. 5 and 20 or Ref. 21 for a diagrammatic derivation. The resulting equation for the one-particle vertex is given by

$$
\frac{\mathrm{d}}{\mathrm{d} \Lambda} \gamma_{1}^{\Lambda}\left(q_{1}^{\prime}, q_{1}\right)=T \sum_{q_{2}^{\prime}, q_{2}} \mathcal{S}_{q_{2}, q_{2}^{\prime}}^{\Lambda} \gamma_{2}^{\Lambda}\left(q_{2}^{\prime}, q_{1}^{\prime} ; q_{2}, q_{1}\right)
$$

where $q_{i}$ is a shorthand for all quantum numbers and the fermionic Matsubara frequency associated with the legs of a vertex, and the full- and single-scale propagators are defined via

$$
\begin{aligned}
\mathcal{G}^{\Lambda} & =\left[\left[\mathcal{G}_{0}^{\Lambda}\right]^{-1}-\Sigma^{\Lambda}\right]^{-1}, \\
\mathcal{S}^{\Lambda} & =\mathcal{G}^{\Lambda} \partial_{\Lambda}\left[\mathcal{G}_{0}^{\Lambda}\right]^{-1} \mathcal{G}^{\Lambda},
\end{aligned}
$$

respectively. The structure of the vertex consists naturally of three different parquet-like channels

$$
\gamma_{2}^{\Lambda}=v+\gamma_{p}^{\Lambda}+\gamma_{x}^{\Lambda}+\gamma_{d}^{\Lambda},
$$

where $v$ is the bare vertex and we refer to $\gamma_{p}^{\Lambda}, \gamma_{x}^{\Lambda}$, and $\gamma_{d}^{\Lambda}$ as the particle-particle channel $(P)$, and the exchange $(X)$ and direct $(D)$ part of the particle-hole channel. These quantities are defined via their flow equations

$$
\frac{\mathrm{d}}{\mathrm{d} \Lambda} \gamma_{2}^{\Lambda}=\frac{\mathrm{d}}{\mathrm{d} \Lambda}\left(\gamma_{p}^{\Lambda}+\gamma_{x}^{\Lambda}+\gamma_{d}^{\Lambda}\right),
$$

and the initial conditions $\gamma_{p}^{\Lambda_{i}}=\gamma_{x}^{\Lambda_{i}}=\gamma_{d}^{\Lambda_{i}}=0$. The explicit form of the flow equations is

$$
\begin{aligned}
\frac{\mathrm{d}}{\mathrm{d} \Lambda} \gamma_{p}^{\Lambda}\left(q_{1}^{\prime}, q_{2}^{\prime} ; q_{1}, q_{2}\right) & =T \sum_{q_{3}^{\prime}, q_{3}, q_{4}^{\prime}, q_{4}} \gamma_{2}^{\Lambda}\left(q_{1}^{\prime}, q_{2}^{\prime} ; q_{3}, q_{4}\right) \mathcal{S}_{q_{3}, q_{3}^{\prime}}^{\Lambda} \mathcal{G}_{q_{4}, q_{4}^{\prime}}^{\Lambda} \gamma_{2}^{\Lambda}\left(q_{3}^{\prime}, q_{4}^{\prime} ; q_{1}, q_{2}\right) \\
\frac{\mathrm{d}}{\mathrm{d} \Lambda} \gamma_{x}^{\Lambda}\left(q_{1}^{\prime}, q_{2}^{\prime} ; q_{1}, q_{2}\right) & =T \sum_{q_{3}^{\prime}, q_{3}, q_{4}^{\prime}, q_{4}} \gamma_{2}^{\Lambda}\left(q_{1}^{\prime}, q_{4}^{\prime} ; q_{3}, q_{2}\right)\left[\mathcal{S}_{q_{3}, q_{3}^{\prime}}^{\Lambda} \mathcal{G}_{q_{4}, q_{4}^{\prime}}^{\Lambda}+\mathcal{G}_{q_{3}, q_{3}^{\prime}}^{\Lambda} \mathcal{S}_{q_{4}, q_{4}^{\prime}}^{\Lambda}\right] \gamma_{2}^{\Lambda}\left(q_{3}^{\prime}, q_{2}^{\prime} ; q_{1}, q_{4}\right) \\
\frac{\mathrm{d}}{\mathrm{d} \Lambda} \gamma_{d}^{\Lambda}\left(q_{1}^{\prime}, q_{2}^{\prime} ; q_{1}, q_{2}\right) & =-T \sum_{q_{3}^{\prime}, q_{3}, q_{4}^{\prime}, q_{4}} \gamma_{2}^{\Lambda}\left(q_{1}^{\prime}, q_{3}^{\prime} ; q_{1}, q_{4}\right)\left[\mathcal{S}_{q_{4}, q_{4}^{\prime}}^{\Lambda} \mathcal{G}_{q_{3}, q_{3}^{\prime}}^{\Lambda}+\mathcal{G}_{q_{4}, q_{4}^{\prime}}^{\Lambda} \mathcal{S}_{q_{3}, q_{3}^{\prime}}^{\Lambda}\right] \gamma_{2}^{\Lambda}\left(q_{4}^{\prime}, q_{2}^{\prime} ; q_{3}, q_{2}\right)
\end{aligned}
$$


feedback between channels by the feedback length $L$.

\section{A. Frequency Parametrisation}

Since we have energy conservation at each vertex,

$$
\begin{aligned}
& \gamma_{1}\left(q_{1}^{\prime}, q_{1}\right) \propto \delta_{n_{1}^{\prime} n_{1}}, \\
& \gamma_{2}\left(q_{1}^{\prime}, q_{2}^{\prime} ; q_{1}, q_{2}\right) \propto \delta_{n_{1}^{\prime}+n_{2}^{\prime} n_{1}+n_{2}},
\end{aligned}
$$

we can parametrize the frequency dependence of the selfenergy with one frequency, and of the vertex with three frequencies. A detailed discussion of the frequency structure is given in Refs. 4, 8, and 9, and since we proceed analogously, we will be very brief here. A convenient choice for the parametrization of the vertex frequency structure is given in terms of the three bosonic frequencies $[7]$

$$
\begin{aligned}
& \Pi=\omega_{n_{1}^{\prime}}+\omega_{n_{2}^{\prime}}=\omega_{n_{1}}+\omega_{n_{2}}, \\
& \mathrm{X}=\omega_{n_{1}^{\prime}}-\omega_{n_{2}}=\omega_{n_{1}}-\omega_{n_{2}^{\prime}}, \\
& \Delta=\omega_{n_{1}^{\prime}}-\omega_{n_{1}}=\omega_{n_{2}}-\omega_{n_{2}^{\prime}} .
\end{aligned}
$$

In order to keep notation short, the frequency information is separated from the site and spin quantum numbers:

$$
\begin{aligned}
& \gamma_{2}\left(j_{1}^{\prime} \sigma_{1}^{\prime} \omega_{n_{1}^{\prime}}, j_{2}^{\prime} \sigma_{2}^{\prime} \omega_{n_{2}^{\prime}} ; j_{1} \sigma_{1} \omega_{n_{1}}, j_{2} \sigma_{2} \omega_{n_{2}}\right) \\
& \quad=\delta_{n_{1}^{\prime}+n_{2}^{\prime} n_{1}+n_{2}} \gamma_{2}\left(j_{1}^{\prime} \sigma_{1}^{\prime}, j_{2}^{\prime} \sigma_{2}^{\prime} ; j_{1} \sigma_{1}, j_{2} \sigma_{2} ; \Pi, \mathrm{X}, \Delta\right)
\end{aligned}
$$

For convenience, we have here also listed the fermionic frequencies in terms of the bosonic ones:

$$
\begin{array}{ll}
\omega_{n_{1}^{\prime}}=\frac{1}{2}(\Pi+\mathrm{X}+\Delta), & \omega_{n_{2}^{\prime}}=\frac{1}{2}(\Pi-\mathrm{X}-\Delta), \\
\omega_{n_{1}}=\frac{1}{2}(\Pi+\mathrm{X}-\Delta), & \omega_{n_{2}}=\frac{1}{2}(\Pi-\mathrm{X}+\Delta) .
\end{array}
$$

\section{B. Coupled-Ladder Approximation}

The basic idea of the CLA scheme was introduced in Refs. 4 and 9 for the frequency parametrization of the single-impurity Anderson model and was further developed for inhomogeneous Fermi systems with onsite interaction in Ref. 7. Here we will go one step further and extend this scheme to treat interacting models with two-particle interactions of finite range, using an idea similar to the singular mode fRG approach introduced in 22. There, the vertex structure in momentum space was decomposed into fermion bilinears that interact via exchange bosons and it was shown that this decomposition admits a systematic approximation by an expansion using form factors. Here, we will proceed similar in position space, introducing "short indices" $k, l$ that will control the extent of our approximation and act similar to the mentioned form factor expansion.

In the case of third-order truncated fRG, BHD introduced two different approximation schemes. The simpler "static second order fRG" (sfRG2) neglects the frequency dependence of the vertex; the more elaborate "dynamic second order fRG" (dfRG2) includes the frequency dependence of the vertex within a channel approximation, reducing this dependence from the generic $\mathcal{O}\left(N_{f}^{3}\right)$ to $\mathcal{O}\left(N_{f}\right)$ where $N_{f}$ is the number of used frequencies. In the case of the onsite model, it turned out that static compared to dynamic fRG produces some artifacts concerning the pinch-off point of the conductance of a QPC, but yields essentially the same shape for the conductance steps as dynamic fRG. For this reason and since it is a factor of $N_{f}$ cheaper, we will only compute the static fRG flow in our numerical work. Nevertheless, we will derive here the full dynamic flow equations, and in principle, it should be no problem to implement these too.

The dfRG2 scheme exploits the fact that the bare vertex consists of a density-density interaction

$$
\begin{aligned}
& v\left(j_{1}^{\prime} \sigma_{1}^{\prime}, j_{2}^{\prime} \sigma_{2}^{\prime} ; j_{1} \sigma_{1}, j_{2} \sigma_{2}\right)= \\
& \quad \delta_{j_{1} j_{2}}^{L_{U}} U_{j_{1} j_{2}}\left[\left(1-\delta_{j_{1} j_{2}}\right) \delta_{\sigma_{1} \sigma_{2}}+\delta_{\sigma_{1} \bar{\sigma}_{2}}\right] \\
& \quad \times\left(\delta_{j_{1}^{\prime} j_{1}} \delta_{j_{2}^{\prime} j_{2}} \delta_{\sigma_{1}^{\prime} \sigma_{1}} \delta_{\sigma_{2}^{\prime} \sigma_{2}}-\delta_{j_{1}^{\prime} j_{2}} \delta_{j_{2}^{\prime} j_{1}} \delta_{\sigma_{1}^{\prime} \sigma_{2}} \delta_{\sigma_{2}^{\prime} \sigma_{1}}\right)
\end{aligned}
$$

and parametrizes the vertex in terms of $\mathcal{O}\left(N^{2} L_{U}^{2} N_{\mathrm{f}}\right)$ independent variables. Here $\delta_{j_{1} j_{2}}^{L_{U}}=1$ if $\left|j_{1}-j_{2}\right| \leq L_{U}$ and is otherwise set to zero.

Using this vertex, we can now consider a simplified version of the vertex flow equation (12), where the feedback of the vertex flow is neglected: on the r.h.s. we replace $\gamma_{2}^{\Lambda} \rightarrow v$. If the feedback of the self-energy were also neglected, this would be equivalent to calculating the vertex in second order perturbation theory. As a consequence, all generated vertex contributions have one of the following structures:

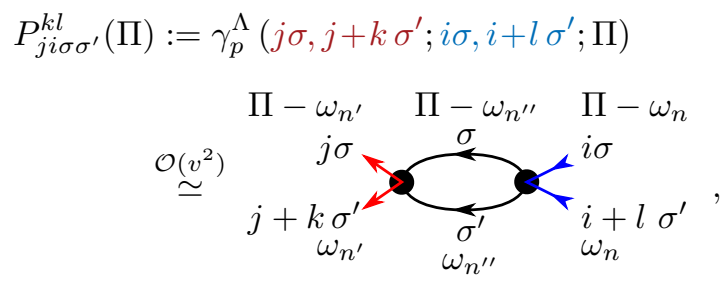

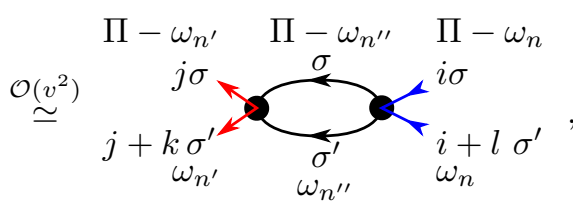

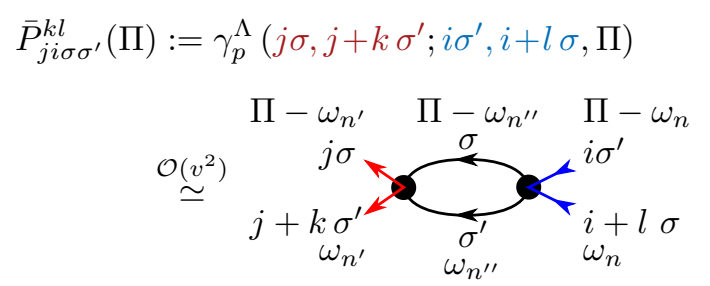

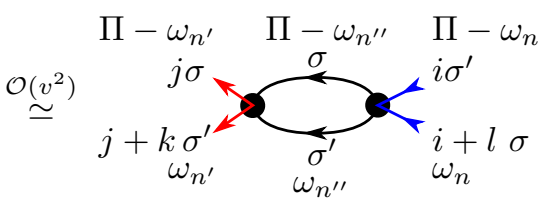

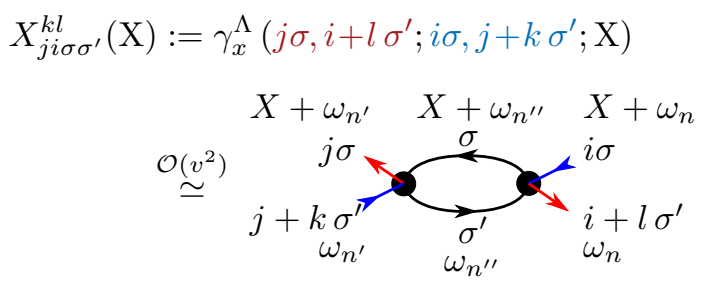

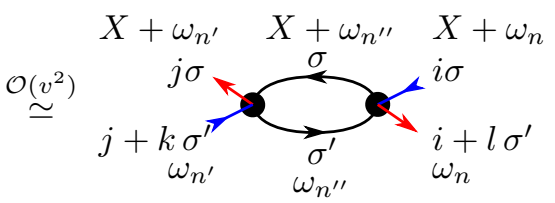

$$
\begin{aligned}
& \bar{X}_{j i \sigma \sigma^{\prime}}^{k l}(\mathrm{X}):=\gamma_{x}^{\Lambda}\left(j \sigma, i+l \sigma^{\prime} ; i \sigma^{\prime}, j+k \sigma ; \mathrm{X}\right) \\
& \stackrel{\mathcal{O}\left(v^{2}\right)}{\simeq} \underset{j+k \sigma_{\omega_{n^{\prime}}}}{j \sigma} \underbrace{X+\omega_{n^{\prime \prime}}}_{\substack{\omega_{n^{\prime \prime}} \\
\omega_{\omega_{n}}}} \begin{array}{l}
X+\omega_{n} \\
i \sigma^{\prime}
\end{array}
\end{aligned}
$$




$$
\begin{aligned}
& D_{j i \sigma \sigma^{\prime}}^{k l}(\Delta):=\gamma_{d}^{\Lambda}\left(j \sigma, i+l \sigma^{\prime} ; j+k \sigma, i \sigma^{\prime} ; \Delta\right)
\end{aligned}
$$

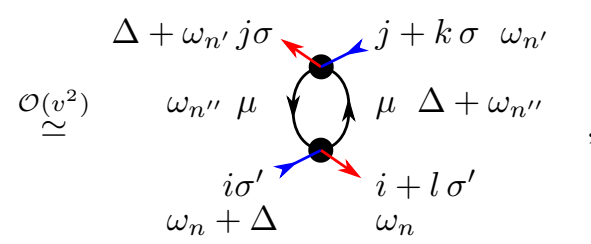

$$
\begin{aligned}
& \bar{D}_{j i \sigma \sigma^{\prime}}^{k l}(\Delta):=\gamma_{d}^{\Lambda}\left(j \sigma, i+l \sigma^{\prime} ; j+k \sigma^{\prime}, i \sigma ; \Delta\right)
\end{aligned}
$$

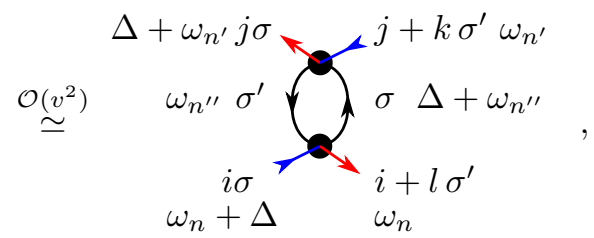

These terms depend only on a single bosonic frequency. The upper indices ${ }^{k l}$, are taken to run over the range

$$
-L \leq k, l \leq L
$$

where the control parameter $L$ sets the "spatial feedback range". The bounds on the lower indices depend on the upper indices: if one of the site indices of $\gamma_{2}$ lies outside the region $\left[-N^{\prime}, N^{\prime}\right]$ where $N^{\prime}$ is defined by $N=2 N^{\prime}+1$, $\gamma_{2}$ is zero. Therefore, $i, j$ run between

$$
\begin{aligned}
\max \left(-N^{\prime},-N^{\prime}-l\right) & \leq i \leq \min \left(N^{\prime}, N^{\prime}-l\right) \\
\max \left(-N^{\prime},-N^{\prime}-k\right) & \leq j \leq \min \left(N^{\prime}, N^{\prime}-k\right)
\end{aligned}
$$

Analogously to BHD, we now feed back all those terms on the r.h.s. of the flow equation (12) which conserve the site and spin structure indicated in Eq. (18). As a first consequence, each vertex quantity is fully fed back into its own flow equation. Secondly, the feedback between different quantities is restricted to those site indices which have the appropriate structure. Furthermore, to avoid frequency mixing, the feedback to a given channel from the other two channels is restricted to using only the static, i.e. zero frequency component of the latter.

This scheme can be expressed by the replacement

$$
\gamma_{2} \rightarrow \tilde{\gamma}_{a}
$$

on the r.h.s. of channel $a=p, x, d$ in Eq. (12) where $\tilde{\gamma}_{a}$ is defined as:

$$
\begin{aligned}
& \tilde{\gamma}_{p}\left(j_{1}^{\prime} \sigma_{1}^{\prime}, j_{2}^{\prime} \sigma_{2}^{\prime} ; j_{1} \sigma_{1}, j_{2} \sigma_{2}, \Pi\right) \\
& \quad=\delta_{j_{1}^{\prime} j_{2}^{\prime}}^{L} \delta_{j_{1} j_{2}}^{L} \gamma_{2}\left(j_{1}^{\prime} \sigma_{1}^{\prime}, j_{2}^{\prime} \sigma_{2}^{\prime} ; j_{1} \sigma_{1}, j_{2} \sigma_{2} ; \Pi, 0,0\right) \\
& \tilde{\gamma}_{x}\left(j_{1}^{\prime} \sigma_{1}^{\prime}, j_{2}^{\prime} \sigma_{2}^{\prime} ; j_{1} \sigma_{1}, j_{2} \sigma_{2}, \mathrm{X}\right)
\end{aligned}
$$

$$
\begin{aligned}
& \quad=\delta_{j_{1}^{\prime} j_{2}}^{L} \delta_{j_{2}^{\prime} j_{1}}^{L} \gamma_{2}\left(j_{1}^{\prime} \sigma_{1}^{\prime}, j_{2}^{\prime} \sigma_{2}^{\prime} ; j_{1} \sigma_{1}, j_{2} \sigma_{2} ; 0, \mathrm{X}, 0\right) \\
& \tilde{\gamma}_{d}\left(j_{1}^{\prime} \sigma_{1}^{\prime}, j_{2}^{\prime} \sigma_{2}^{\prime} ; j_{1} \sigma_{1}, j_{2} \sigma_{2}, \Delta\right) \\
& \quad=\delta_{j_{1}^{\prime} j_{1}}^{L} \delta_{j_{2}^{\prime} j_{2}}^{L} \gamma_{2}\left(j_{1}^{\prime} \sigma_{1}^{\prime}, j_{2}^{\prime} \sigma_{2}^{\prime} ; j_{1} \sigma_{1}, j_{2} \sigma_{2} ; 0,0, \Delta\right)
\end{aligned}
$$

\section{Symmetries}

As can readily be checked, these flow equations respect the following symmetry relations:

$$
\begin{aligned}
\mathcal{G}_{i j}^{\sigma \Lambda}\left(\omega_{n}\right) & =\mathcal{G}_{j i}^{\sigma \Lambda}\left(\omega_{n}\right)=\left[\mathcal{G}_{i j}^{\sigma \Lambda}\left(-\omega_{n}\right)\right]^{*}, \\
\Sigma_{i j}^{\sigma \Lambda}\left(\omega_{n}\right) & =\Sigma_{j i}^{\sigma \Lambda}\left(\omega_{n}\right)=\left[\Sigma_{i j}^{\sigma \Lambda}\left(-\omega_{n}\right)\right]^{*}, \\
P_{j i \sigma \sigma^{\prime}}^{k l}(\Pi) & =P_{i j \sigma \sigma^{\prime}}^{l k}(\Pi)=P_{(j+k)(i+l) \sigma^{\prime} \sigma}^{(-k)(-l)}(\Pi), \\
\bar{P}_{j i \sigma \sigma^{\prime}}^{k l}(\Pi) & =\bar{P}_{i j \sigma^{\prime} \sigma}^{l k}(\Pi)=\bar{P}_{(j+k)(i+l) \sigma^{\prime} \sigma}^{(-k)(-l)}(\Pi), \\
P_{j i \sigma \sigma^{\prime}}^{k l}(\Pi) & =-\bar{P}_{j+k i \sigma^{\prime} \sigma}^{-k l}(\Pi)=-\bar{P}_{j(i+l) \sigma \sigma^{\prime}}^{k(-l)}(\Pi), \\
P_{\sigma \sigma} & =\bar{P}_{\sigma \sigma},
\end{aligned}
$$

$$
\begin{aligned}
X_{j i \sigma \sigma^{\prime}}^{k l}(X) & =X_{i j \sigma \sigma^{\prime}}^{l k}(X)=\left[X_{(j+k)(i+l) \sigma^{\prime} \sigma}^{(-k)(-l)}(X)\right]^{*}, \\
\bar{X}_{j i \sigma \sigma^{\prime}}^{k l}(X) & =\bar{X}_{i j \sigma^{\prime} \sigma}^{l k}(X)=\left[\bar{X}_{(j+k)(i+l) \sigma \sigma^{\prime}}^{(-k)(-l)}(X)\right]^{*} \\
X_{\sigma \sigma} & =\bar{X}_{\sigma \sigma}
\end{aligned}
$$

$$
X=-\bar{D}, \quad \bar{X}=-D
$$

$$
\begin{aligned}
P_{j i \sigma \sigma^{\prime}}^{k l}(\Pi) & =\left[P_{j i \sigma \sigma^{\prime}}^{k l}(-\Pi)\right]^{*}, \\
X_{j i \sigma \sigma^{\prime}}^{k l}(\mathrm{X}) & =\left[X_{j i \sigma \sigma^{\prime}}^{k l}(-\mathrm{X})\right]^{*}, \\
\bar{X}_{j i \sigma \sigma^{\prime}}^{k l}(\Delta) & =\left[\bar{X}_{j i \sigma \sigma^{\prime}}^{k l}(-\Delta)\right]^{*}
\end{aligned}
$$

As a result, all relevant information is contained in a small number of independent frequency-dependent blockmatrices, which we define as follows:

$$
\begin{aligned}
& P^{\Lambda}=P_{\uparrow \downarrow}^{\Lambda}, P_{\sigma}^{\Lambda}=P_{\sigma \sigma}^{\Lambda}, \\
& X^{\Lambda}=X_{\uparrow \downarrow}^{\Lambda}, \\
& D^{\Lambda}=D_{\uparrow \downarrow}^{\Lambda}, D_{\sigma}^{\Lambda}=D_{\sigma \sigma}^{\Lambda},
\end{aligned}
$$

where the superscript $\Lambda$ signifies a dependence on the flow parameter.

The flow equations for these matrices can be derived starting from Eqs. (12). The replacement (22) restricts the internal quantum numbers on the r.h.s. of the flow equation $q_{3}, q_{4}, q_{3}^{\prime}$, and $q_{4}^{\prime}$ according to the definitions (18):

$$
\begin{aligned}
\dot{P}_{j i}^{k l \Lambda}(\Pi) & =\dot{\gamma}_{p}^{\Lambda}(j \uparrow, j+k \downarrow ; i \uparrow, i+l \downarrow ; \Pi) \\
& =T \sum_{j^{\prime} i^{\prime} k^{\prime} l^{\prime}, n}\left[\tilde{\gamma}_{p}^{\Lambda}\left(j \uparrow, j+k \downarrow ; i^{\prime} \uparrow, i^{\prime}+l^{\prime} \downarrow ; \Pi\right) \mathcal{S}_{i^{\prime} j^{\prime}}^{\uparrow \Lambda}\left(\omega_{n}\right) \mathcal{G}_{i^{\prime}+l^{\prime} j^{\prime}+k^{\prime}}^{\downarrow \Lambda}\left(\Pi-\omega_{n}\right) \tilde{\gamma}_{p}^{\Lambda}\left(j^{\prime} \uparrow, j^{\prime}+k^{\prime} \downarrow ; i \uparrow, i+l \downarrow ; \Pi\right)\right.
\end{aligned}
$$




$$
\begin{aligned}
& \left.+\tilde{\gamma}_{p}^{\Lambda}\left(j \uparrow, j+k \downarrow ; i^{\prime} \downarrow, i^{\prime}+l^{\prime} \uparrow ; \Pi\right) \mathcal{S}_{i^{\prime} j^{\prime}}^{\downarrow \Lambda}\left(\omega_{n}\right) \mathcal{G}_{i^{\prime}+l^{\prime} j^{\prime}+k^{\prime}}^{\uparrow \Lambda}\left(\Pi-\omega_{n}\right) \tilde{\gamma}_{p}^{\Lambda}\left(j^{\prime} \downarrow, j^{\prime}+k^{\prime} \uparrow ; i \uparrow, i+l \downarrow ; \Pi\right)\right], \\
\dot{P}_{j i \sigma}^{k l \Lambda}(\Pi) & =\dot{\gamma}_{p}^{\Lambda}(j \sigma, j+k \sigma ; i \sigma, i+l \sigma ; \Pi) \\
& =T \sum_{j^{\prime} i^{\prime} k^{\prime} l^{\prime}, n} \tilde{\gamma}_{p}^{\Lambda}\left(j \sigma, j+k \sigma ; i^{\prime} \sigma, i^{\prime}+l^{\prime} \sigma ; \Pi\right) \mathcal{S}_{i^{\prime} j^{\prime}}^{\sigma \Lambda}\left(\omega_{n}\right) \mathcal{G}_{i^{\prime}+l^{\prime} j^{\prime}+k^{\prime}}^{\sigma \Lambda}\left(\Pi-\omega_{n}\right) \tilde{\gamma}_{p}^{\Lambda}\left(j^{\prime} \sigma, j^{\prime}+k^{\prime} \sigma ; i \sigma, i+l \sigma ; \Pi\right) \\
\dot{X}_{j i}^{k l \Lambda}(\mathrm{X})= & \dot{\gamma}_{x}^{\Lambda}(j \uparrow, i+l \downarrow ; i \uparrow, j+k \downarrow ; \mathrm{X}) \\
= & T \sum_{i^{\prime} j^{\prime} l^{\prime} k^{\prime}, n} \tilde{\gamma}_{x}^{\Lambda}\left(j \uparrow, i^{\prime}+l^{\prime} \downarrow ; i^{\prime} \uparrow, j+k \downarrow ; \mathrm{X}\right)\left[\mathcal{S}_{i^{\prime} j^{\prime}}^{\uparrow \Lambda}\left(\omega_{n}+\mathrm{X}\right) \mathcal{G}_{j^{\prime}+k^{\prime} i^{\prime}+l^{\prime}}^{\downarrow \Lambda}\left(\omega_{n}\right)+\mathcal{S}_{j^{\prime}+k^{\prime} i^{\prime}+l^{\prime}}^{\downarrow \Lambda}\left(\omega_{n}\right) \mathcal{G}_{i^{\prime} j^{\prime}}^{\uparrow \Lambda}\left(\omega_{n}+\mathrm{X}\right)\right] \\
\dot{D}_{j i \sigma \sigma^{\prime}}^{k l \Lambda}(\mathrm{X})= & \dot{\gamma}_{d}^{\Lambda}\left(j \sigma, i+l \sigma^{\prime} ; j+k \sigma, i \sigma^{\prime} ; \Delta\right) \\
= & -T \sum_{i^{\prime} j^{\prime} l^{\prime} k^{\prime}} \tilde{\gamma}_{d}^{\Lambda}\left(j \sigma, i^{\prime}+l^{\prime} \sigma^{\prime \prime} ; j+k \sigma, i^{\prime} \sigma^{\prime \prime} ; \Delta\right)\left[\mathcal{S}_{i^{\prime}+l^{\prime} j^{\prime}+k^{\prime}}^{\sigma^{\prime \prime} \Lambda}\left(\omega_{n}\right) \mathcal{G}_{i^{\prime} j^{\prime}}^{\sigma^{\prime \prime} \Lambda}\left(\omega_{n}+\Delta\right)+\mathcal{G}_{i^{\prime}+l^{\prime} j^{\prime}+k^{\prime}}^{\sigma^{\prime \prime} \Lambda}\left(\omega_{n}\right) \mathcal{S}_{i^{\prime} j^{\prime}}^{\sigma^{\prime \prime} \Lambda}\left(\omega_{n}+\Delta\right)\right] \\
& \tilde{\gamma}_{d}^{\Lambda}\left(j^{\prime} \sigma^{\prime \prime}, i+l \sigma^{\prime} ; j^{\prime}+k^{\prime} \sigma^{\prime \prime}, i \sigma^{\prime} ; \Delta\right)
\end{aligned}
$$

The initial conditions are

$$
P^{\Lambda_{i}}=P_{\sigma}^{\Lambda_{i}}=X^{\Lambda_{i}}=D_{\sigma \sigma^{\prime}}^{\Lambda_{i}}=0 .
$$

These equations can be compactly written in blockmatrix form

$$
\begin{aligned}
& \frac{d}{d \Lambda} P^{\Lambda}(\Pi)=\tilde{P}^{\Lambda}(\Pi) \cdot W^{p \Lambda}(\Pi) \cdot \tilde{P}^{\Lambda}(\Pi) \\
& \frac{d}{d \Lambda} P_{\sigma}^{\Lambda}(\Pi)=\tilde{P}_{\sigma}^{\Lambda}(\Pi) \cdot W_{\sigma}^{p \Lambda}(\Pi) \cdot \tilde{P}_{\sigma}^{\Lambda}(\Pi), \\
& \frac{d}{d \Lambda} X^{\Lambda}(\mathrm{X})=\tilde{X}^{\Lambda}(\mathrm{X}) \cdot W^{x \Lambda}(\mathrm{X}) \cdot \tilde{X}^{\Lambda}(\mathrm{X}),
\end{aligned}
$$

$$
\frac{d}{d \Lambda} D_{\sigma \sigma^{\prime}}^{\Lambda}(\Delta)=-\sum_{\sigma^{\prime \prime}} \tilde{D}_{\sigma \sigma^{\prime \prime}}^{\Lambda}(\Delta) \cdot W_{\sigma^{\prime \prime}}^{d \Lambda}(\Delta) \cdot \tilde{D}_{\sigma^{\prime \prime} \sigma^{\prime}}^{\Lambda}(\Delta),
$$

where ' $'$ ' denotes a block-matrix multiplication:

$$
[A \cdot B]_{j i}^{k l}=\sum_{j^{\prime} k^{\prime}} A_{j j^{\prime}}^{k k^{\prime}} B_{j^{\prime} i}^{k^{\prime} l}
$$

and we have introduced the definitions

$$
\begin{aligned}
\tilde{P}_{j i}^{k l \Lambda}(\Pi) & =\tilde{\gamma}_{p}^{\Lambda}(j \uparrow, j+k \downarrow ; i \uparrow, i+l \downarrow ; \Pi) \\
& =\delta_{j i} \delta_{k l} U_{j j+k}+P_{j i}^{k l \Lambda}(\Pi)+\delta_{j i+l}^{L} \delta_{i j+k}^{L} X_{j i}^{(i+l-j)(j+k-i) \Lambda}(0)+\delta_{i j}^{L} \delta_{j+k i+l}^{L} D_{j(i+l) \uparrow \downarrow}^{(i-j)(j+k-i-l) \Lambda}(0), \\
\tilde{P}_{j i \sigma}^{k l \Lambda}(\Pi) & =\tilde{\gamma}_{p}^{\Lambda}(j \sigma, j+k \sigma ; i \sigma, i+l \sigma ; \Pi) \\
& =\delta_{j i} \delta_{k l} U_{j j+k}-\delta_{k,-l} \delta_{(j+k) i} U_{j i}+P_{j i \sigma}^{k l \Lambda}(\Pi)-\delta_{i+l j}^{L} \delta_{j+k i}^{L} D_{j i \sigma}^{(i+l-j)(j+k-i) \Lambda}(0)+\delta_{i j}^{L} \delta_{j+k i+l}^{L} D_{j(i+l) \sigma}^{(i-j)(j+k-i-l) \Lambda}(0), \\
\tilde{X}_{j i}^{k l \Lambda}(\mathrm{X}) & =\tilde{\gamma}_{x}^{\Lambda}(j \uparrow, i+l \downarrow ; i \uparrow, j+k \downarrow ; \mathrm{X}) \\
& =\delta_{j i} \delta_{k l} U_{j j+k}+X_{j i}^{k l \Lambda}(\mathrm{X})+\delta_{i+l j}^{L} \delta_{j+k i}^{L} P_{j i}^{(i+l-j)(j+k-i) \Lambda}(0)+\delta_{i j}^{L} \delta_{j+k i+l}^{L} D_{j(j+k) \uparrow \downarrow}^{(i-j)(i+l-j-k) \Lambda}(0), \\
\tilde{D}_{j i \sigma \sigma^{\prime}}^{k l \Lambda}(\Delta) & =\tilde{\gamma}_{d}^{\Lambda}\left(j \sigma, i+l \sigma^{\prime} ; j+k \sigma, i \sigma^{\prime} ; \Delta\right) \\
& =\delta_{0 k} \delta_{0 l} U_{j i}-\delta_{\sigma \sigma^{\prime}} \delta_{j i} \delta_{k l} U_{j j+k}+D_{j i \sigma \sigma^{\prime}}^{k l \Lambda}(\Delta)+\delta_{i+l j}^{L} \delta_{j+k i}^{L} P_{j(j+k) \sigma \sigma^{\prime}}^{(i+l-j)(i-j) \Lambda}(0)+\delta_{i j}^{L} \delta_{j+k i+l}^{L} X_{j(j+k) \sigma \sigma^{\prime}}^{(i-j)(i+l-k) \Lambda}(0),
\end{aligned}
$$

which account for the inter-channel feedback contained in equation (22). Note that Eq. (31d) is not fully expressed in terms of the definitions (26). This can only been done once $\sigma$ and $\sigma^{\prime}$ are specified explicitly and then leads to three independent equations. $W^{p}, W^{x}$ and $W^{d}$ each rep- resent a specific bubble, i.e. a product of two propagators summed over an internal frequency:

$$
W_{i j}^{l k, p \Lambda}(\Pi)=T \sum_{n}\left[\mathcal{S}_{i j}^{\uparrow \Lambda}\left(\omega_{n}\right) \mathcal{G}_{i+l j+k}^{\downarrow \Lambda}\left(\Pi-\omega_{n}\right)\right.
$$




$$
\begin{aligned}
& \left.+\mathcal{S}_{i+l j+k}^{\downarrow \Lambda}\left(\omega_{n}\right) \mathcal{G}_{i j}^{\uparrow \Lambda}\left(\Pi-\omega_{n}\right)\right] \\
W_{i j \sigma}^{l k, p \Lambda}(\Pi)=T \sum_{n}[ & \left.\mathcal{S}_{i j}^{\sigma \Lambda}\left(\omega_{n}\right) \mathcal{G}_{i+l j+k}^{\sigma \Lambda}\left(\Pi-\omega_{n}\right)\right] \\
W_{i j}^{l k, x \Lambda}(\mathrm{X})=T \sum_{n}[ & \mathcal{S}_{i+l j+k}^{\downarrow \Lambda}\left(\omega_{n}\right) \mathcal{G}_{i j}^{\uparrow \Lambda}\left(\omega_{n}+\mathrm{X}\right) \\
+ & \left.\mathcal{G}_{j+k i+l}^{\downarrow \Lambda}\left(\omega_{n}\right) \mathcal{S}_{i j}^{\uparrow \Lambda}\left(\omega_{n}+\mathrm{X}\right)\right], \\
W_{i j \sigma}^{l k, d \Lambda}(\Delta)=T \sum_{n}[ & \mathcal{S}_{i+l j+k}^{\sigma \Lambda}\left(\omega_{n}\right) \mathcal{G}_{i j}^{\sigma \Lambda}\left(\omega_{n}+\Delta\right) \\
& \left.+\mathcal{G}_{i+l j+k}^{\sigma \Lambda}\left(\omega_{n}\right) \mathcal{S}_{i j}^{\sigma \Lambda}\left(\omega_{n}+\Delta\right)\right] .
\end{aligned}
$$

\section{D. eCLA vs. CLA and the Role of $D_{\uparrow \downarrow}$}

Let us now recapitulate the similarities and differences between our new eCLA method to the previous CLA method used in Ref. 8. There, only onsite models were considered and the guiding idea for approximations in the fRG flow was to include only those vertex structures which are already generated in second order in the interaction. Therefore it was sufficient to consider only an onsite feedback between the individual channels, i.e. the feedback range was the same as the interaction range. In the development of the eCLA we followed the same idea, but found it to be advantagous to separate the feedback length $L$ from the actual range of the interaction $L_{U}$. To be exact in second order, $L$ has to be chosen at least as large as $L_{U}$. However, it can be chosen also larger than $L_{U}$, and thus enables us to study the importance of the neglected higher order terms. If $L$ is chosen exactly equal to $L_{U}$, we are in principle back at the original idea to include only vertex structures in the flow which are already generated in second order of the interaction.

However, there is one exception to the last statement: for purely onsite interactions $\left(L_{U}=0\right)$, the contributions of $D^{\uparrow \downarrow}$ and $P^{\sigma \sigma}$ to the vertex are of third and fourth order, respectively. In Ref. 8 they were therefore neglected, consistent with the policy of keeping only structures generated in second order. In the present paper, however, our implementation does not explicitly distinguish between $L_{U}=0$ and $L_{U}>0$ and includes the $D^{\uparrow \downarrow}$ and $P^{\sigma \sigma}$ contributions regardless of the values of $L_{U}$ and $L$, even for $L_{U}=L=0$. To be specific, for $L_{U}=L=0$ our present flow scheme keeps $P^{\sigma \sigma}=0$ but leads to a finite contribution of $D^{\uparrow \downarrow}$. Consequently, our results for $L_{U}=L=0$ differ slightly from those obtained in Refs. 7 and 8 , and the difference is a measure of the magnitude of the third-order $D^{\uparrow \downarrow}$ contribution.

In Fig. 1, we compare the dependence of the QPC conductance on the magnetic field for a model with purely onsite interactions (defined in Sec. III below) for both CLA and eCLA with $L=0$. The difference is most noticeable for $B=0$ in the region of the 0.7 anomly, i.e. in the regime where interactions influence the conductance most strongly, but even here the difference is not very big. (Of course this holds only in intermediate parameter regimes, i.e. in regimes where both the eCLA and the

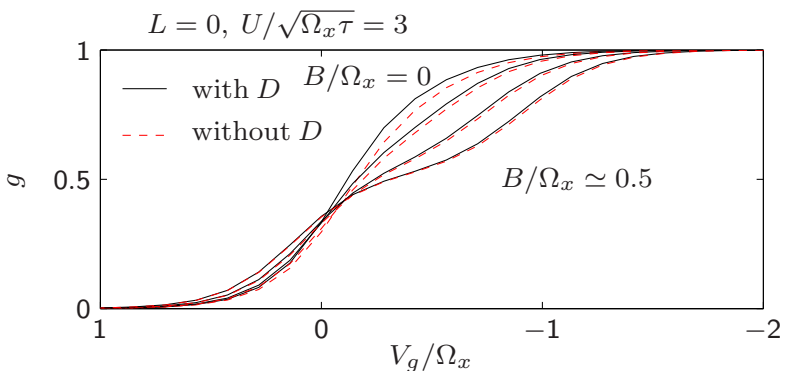

Figure 1. The linear conductance $g=G / G_{Q}$ of a QPC as function of gate voltage, plotted for the cases with and without feedback of $D^{\uparrow \downarrow}$ in an intermediate parameter regime for four equidistant magnetic fields. Note that the difference between the two cases is suppressed with increasing the magnetic field.

CLA are convergent.)

\section{E. The flow equation of the self-energy}

Using the above definitions, the flow equation of the self-energy, (8), can be written explicitly as

$$
\begin{aligned}
\frac{d}{d \Lambda} \Sigma_{j i}^{\sigma \Lambda}\left(\omega_{n}\right)=- & T \sum_{k, \sigma^{\prime}, n^{\prime}}\left\{\sum _ { l } \mathcal { S } _ { i + l , j + k } ^ { \sigma ^ { \prime } \Lambda } ( \omega _ { n } ^ { \prime } ) \left[U_{i(i+l)} \delta_{l k} \delta_{j i}\right.\right. \\
& -U_{i j} \delta_{k,-l} \delta_{j(i+l)} \delta_{\sigma \sigma^{\prime}}+P_{j i \sigma \sigma^{\prime}}^{k l \Lambda}\left(\omega_{n}+\omega_{n}^{\prime}\right) \\
& \left.+X_{j i \sigma \sigma^{\prime}}^{k l \Lambda}\left(\omega_{n}-\omega_{n}^{\prime}\right)\right] \\
& \left.+\sum_{i_{2}} \mathcal{S}_{i_{2}, i_{2}+k}^{\sigma^{\prime} \Lambda}\left(\omega_{n}^{\prime}\right) D_{j i_{2} \sigma \sigma^{\prime}}^{(i-j) k \Lambda}(0)\right\}
\end{aligned}
$$

where the $l, k$-summation is restricted to $|l|,|k| \leq L$, whereas the sum over $i_{2}$ runs over the whole interacting region. To summarize, dfRG2 is defined by the flow equations (29) and (33), together with the definitions (9), (18), (26), (31) and (32).

\section{F. Restrictions for actual computations}

In our actual computations, we restrict ourself to the case of zero temperature and use so called static fRG, meaning that we treat the vertices as frequency independent. The zero-temperature limit enables us to transform the summation over discrete Matsubara frequencies into continuous integrals along the imaginary axis, and the $\Theta_{T}$ in Eq. (7) is a sharp step function. Using this, we are able to apply Morris' lemma [23], which enables us to simplify the integral expressions containing the singlescale propagator $\mathcal{S}$ in the flow equations (27): under integration over $\omega$, the following relations hold:

$$
\begin{aligned}
& \mathcal{S}^{\Lambda}(i \omega) \stackrel{T \equiv 0}{=} \delta(|\omega|-\Lambda) \widetilde{\mathcal{G}}^{\Lambda}(i \omega), \\
& \widetilde{\mathcal{G}}^{\Lambda}(i \omega)=\left[\left[\mathcal{G}_{0}(i \omega)\right]^{-1}-\Sigma^{\Lambda}(i \omega)\right]^{-1},
\end{aligned}
$$




$$
\begin{array}{r}
\mathcal{S}_{i, j}^{\Lambda}\left(i \omega_{1}\right) \mathcal{G}_{k, l}^{\Lambda}\left(i \omega_{2}\right) \stackrel{T=0}{=} \delta\left(\left|\omega_{1}\right|-\Lambda\right) \Theta\left(\left|\omega_{2}\right|-\Lambda\right) \\
\widetilde{\mathcal{G}}_{i, j}^{\Lambda}\left(i \omega_{1}\right) \widetilde{\mathcal{G}}_{k, l}^{\Lambda}\left(i \omega_{2}\right) .
\end{array}
$$

The static fRG approximation treats the vertex quantities $\gamma_{p}^{\Lambda}, \gamma_{x}^{\Lambda}, \gamma_{d}^{\Lambda}$ as frequency independent, setting the bosonic frequencies $\Pi, X, \Delta$ to zero. Via Eq. (8), this automatically implies that the self-energy is frequency independent, too. In the case of QPC models with onsite interaction this approximation was compared with results of the frequency dependent fRG-scheme, so called "dynamic fRG" and was seen to yield reasonable results for the zero-frequency Green's function at zero temperature. However, for models with finite-ranged interactions we find more pronounced static fRG artifacts (described in section IV) which might be improved by the use of the dynamical method. This is a topic for future research. We stress here that it should in principle be straightforward to implement the dynamical method. The main restriction is simply the effort in computation time, which scales like the number of used frequencies, $N_{f}$, which in Ref. 7 is typically of the order $10^{2}$.

\section{G. Numerical implementation}

In a numerical implementation, the flow will start at a value $\Lambda_{i}$ which is usually chosen as large, but is not infinite. For $\Lambda_{i}$ large enough, one can show [5] that the flow of the self-energy from $\Lambda=\infty$ to $\Lambda=\Lambda_{i}$ results in a value of $\gamma_{1}^{\Lambda_{i}}$ given by

$$
\gamma_{1}^{\Lambda_{i}}\left(q_{1}^{\prime}, q_{1}\right)=-\frac{1}{2} \sum_{q} v\left(q, q_{1}^{\prime} ; q, q_{1}\right)
$$

This is then used as the initial condition for $\gamma_{1}$ in the numeric fRG flow. The initial condition for the vertex $\gamma_{2}$, given by Eq. (3), stays the same.

In the case of sfRG2 the vertices and the self-energy only depend on $\Lambda$. In order to carry out the resulting integration, we mapped the domain of the flow parameter $\Lambda \in[0, \infty)$ onto the finite domain $x \in[0,1)$ by using the substitution $\Lambda=\frac{x}{1-x}$, c.f. Ref. 8. To integrate the resulting flow, we followed Dormand-Prince [24], using a 4-th order Runge-Kutta method with adaptive step-size control.

For static fRG, the computationally most expensive step is the block-matrix multiplication of Eq. (29), which scales as $\mathcal{O}\left(N^{3} L^{3}\right)$. In dynamic fRG schemes with nonfrequency cutoff (e.g. with hybridisation flow [4]), for intermediate $N \lesssim 10^{2}$ most of the calculation time is spent on the bubble integrals of Eq. (32), whose calculation time scales as $\mathcal{O}\left(N^{2} L^{2} N_{f}\right)$, where $N_{f}$ is the number of bosonic frequencies. Since the numerical cost for this calculation (for the system sizes used in our setup) is comparable to the block-matrix multiplication of Eq. (29), it might be possible to implement the eCLA within those schemes, too.

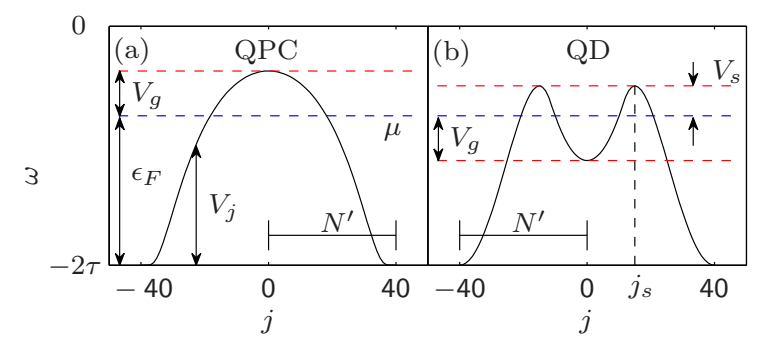

Figure 2. Typical QPC and QD barrier shapes, controlled via the parameters, $\varepsilon_{F}, V_{g}, N^{\prime}$, and, for the $\mathrm{QD}, V_{s}$ and $j_{s}$. For these plots, both $\mu$ and the barrier top lie were chosen to lie below the center of the bulk band, which we take as reference energy where $\omega=0$. The case of half-filled leads, used for most of our calculations, corresponds to choosing $\mu=0$.

\section{RESULTS: ONSITE-INTERACTIONS}

Having derived our eCLA scheme in the last section, we are now able to apply it to the two models of primary interest here, namely the QPC and the QD. In the present section, we study purely onsite models

$$
U_{i j}=\delta_{i j} U
$$

where we treat the strength $U$ of the interaction as a tunable and space-independent parameter, which is suppressed smoothly to zero at the ends of the interacting region. The focus of this section lies on comparing our results to the ones obtained previously by BHD to explore the consequences of the improved feedback for a well-studied example. If not otherwise specified, plots in this section are calculated with $\mu=0$, i.e. with half-filled leads.

\section{A. Models for QPC and QD}

Our interest lies in the low energy physics of a QPC or a QD. For this reason, we consider only the lowest subband of a QPC, or a QD coupled to one-dimensional leads. We use a one-dimensional model Hamiltonian of the same form as used in Refs. [7, 8, 19]:

$$
\hat{H}=\sum_{j \sigma}\left[E_{j}^{\sigma} \hat{n}_{j \sigma}-\tau\left(d_{j \sigma}^{\dagger} d_{j+1 \sigma}+\text { h.c. }\right)\right]+\sum_{j} U_{j} \hat{n}_{j \uparrow} \hat{n}_{j \downarrow} .
$$

It describes an infinite tight-binding chain with constant lattice spacing $a$, constant hopping amplitude $\tau$, onsite interaction $U_{j}$, and on-site potential energy $E_{j}^{\sigma}=$ $V_{j}-\frac{\sigma B}{2}$. Here $V_{j}$ will be used to model the smooth electrostatic QPC or QD potential defined by gates (as described below and illustrated in Fig. 2), and the Zeeman energy $B$ accounts for a uniform external magnetic field parallel to the 2DEG. We take $U_{j}$ and $V_{j}$ to be nonzero only within a (single or double) "barrier region" of $N=2 N^{\prime}+1$ sites centered around $j=0$, containing the $\mathrm{QPC}$ or $\mathrm{QD}$. The rest of the chain represents 
two noninteracting leads with bandwidth $4 \tau$, chemical potential $\mu$, bulk Fermi energy $\varepsilon_{F}=2 \tau+\mu$ and effective mass $m^{*}=\hbar^{2} /\left(2 \tau a^{2}\right)$ (defined as the curvature of the dispersion at the band bottom in the bulk). Adopting the convention in Ref. 8, we choose the center of the bulk band as energy origin. In order to arrive at a discrete QPC potential $V_{j}$, we start with a continuous QPC potential

$$
V(x)= \begin{cases}\left(V_{g}+\varepsilon_{F}\right) \exp \left(\frac{-\gamma^{2}\left(x / L_{\mathrm{bar}}\right)^{2}}{1-\left(x / L_{\mathrm{bar}}\right)^{2}}\right), & |x| \leq L_{\mathrm{bar}} \\ 0 & |x|>L_{\mathrm{bar}}\end{cases}
$$

where $2 L_{\text {bar }}$ is the whole barrier length and $V_{g}$ controls the barrier height, measured w.r.t. $\varepsilon_{F}$. Near the barrier top, the potential (38) can be expanded as

$$
V(x)=V_{g}+\varepsilon_{F}-\frac{1}{2} \frac{m^{*}}{\hbar^{2}} \Omega_{x}^{2} x^{2}+\mathcal{O}\left(x^{4}\right),
$$

where the curvature parameter $\Omega_{x}$ is given by

$$
\Omega_{x}=\gamma \frac{\hbar}{L_{\mathrm{bar}}} \sqrt{\frac{2\left(V_{g}+\varepsilon_{F}\right)}{m^{*}}} .
$$

It has units of energy and serves as characteristic energy scale for the QPC. It also defines a characteristic length scale for the QPC barrier top

$$
l_{x}=\hbar / \sqrt{2 m^{*} \Omega_{x}}=a \sqrt{\tau / \Omega_{x}} .
$$

The dimensionless parameter $\gamma$ in the exponent of Eq. (38) can be used to vary the barrier curvature [Eq. (40)] without changing the barrier height. Through most of section III, we will keep $\gamma=1$ constant and consider only gate-voltages small compared to $\varepsilon_{F}$, such that the curvature can be assumed to be independent of $V_{g}$. However, when studying eCLA convergence properties (Fig. 4), and when dealing with longer-ranged interactions in Sec. IV, we will need to choose $\gamma \neq 1$.

We discretize the QPC potential (38) by choosing a number of sites $N$ and setting the lattice spacing $a=$ $2 L_{\mathrm{bar}} / N$, to arrive at

$$
V_{j}=V(j \cdot a)= \begin{cases}\left(V_{g}+\varepsilon_{F}\right) e^{-\gamma^{2} \frac{\left(j / N^{\prime}\right)^{2}}{1-\left(j / N^{\prime}\right)^{2}},}, & |j| \leq N^{\prime}, \\ 0, & |j|>N^{\prime} .\end{cases}
$$

The resulting barrier shape given by Eq. (42) is plotted in Fig. 2(a). The leading behavior around the maximum at $j=0$ is quadratic and the same as in Ref. 8:

$$
V_{j}=V_{g}+\varepsilon_{F}-\frac{\Omega_{x}^{2}}{4 \tau} j^{2}+\mathcal{O}\left(j^{4}\right),
$$

and the curvature can be expressed through the discrete quantities as $\Omega_{x}=\gamma \frac{2 \sqrt{\tau\left(\varepsilon_{F}+V_{g}\right)}}{N^{\prime}}$. For our onsite studies, where $V_{g}$ is only varied in a small region around $V_{g}=0$, we use the approximation $\Omega_{x}=\gamma \frac{2 \sqrt{\tau \varepsilon_{F}}}{N^{\prime}}$. In order to avoid discretization artifacts, the discretization length $a$ should be chosen significantly smaller than $l_{x}$. In our actual computations for the QPC with onsite interactions we use a ratio $l_{x} / a$ varying between approximately $4-10$ sites.

To model a $\mathrm{QD}$, we use a potential that can be tuned smoothly from the QPC shape described above to a double-barrier structure, as shown in Fig. 2(b). The discretization procedure is analogous to the QPC and we state here only the resulting discrete dot potential, which is the same as used in Refs. 7 and 19:

$$
V_{j}=\left\{\begin{array}{rr}
0, & \forall|j| \geq N^{\prime}, \\
\left(V_{s}+\varepsilon_{F}\right)\left[2\left(\frac{|j|-N^{\prime}}{j_{s}-N^{\prime}}\right)^{2}-\left(\frac{|j|-N^{\prime}}{j_{s}-N^{\prime}}\right)^{4}\right], & \\
& \forall j_{0} \leq|j| \leq N^{\prime}, \\
V_{g}+\varepsilon_{F}+\frac{\bar{\Omega}_{x}^{2} j^{2}}{4 \tau} \operatorname{sgn}\left(V_{s}-V_{g}\right), & \forall 0 \leq|j|<j_{0} .
\end{array}\right.
$$

We can vary the dot width via $j_{s}$, and the depth of the quadratic well in the middle via $V_{s}$ and $V_{g}$. These choices determine the values of $j_{0}$ and $\bar{\Omega}_{x}$ in order to make the potential continuously differentiable. Of course, this is just one convenient way to model the dot structure, and the qualitative behavior of the physical results does not depend on the specific implementation.

For the onsite interaction we use both for the QPC and the QD the form used by BHD [7]:

$$
U_{j}=U e^{-\left(j / N^{\prime}\right)^{6} /\left[1-\left(j / N^{\prime}\right)^{2}\right]} .
$$

It is almost constant and equal to $U$ in the center of the QPC and drops smoothly to zero at the flanks of the barrier region.

\section{B. Physical behavior of the models}

We now briefly summarize the physics of these models, which was already discussed in great detail by BHD in Refs. 7 and 19. Our main handle for tuning the QPC potential is the gate voltage $V_{g}$, which controls the height of the barrier. If the barrier top lies well above the chemical potential, the QPC is closed. Lowering the barrier, the QPC opens up and the linear conductance $g$ increases smoothly from 0 to 1 in the region of gate voltages $0 \lesssim V_{g} \lesssim \Omega_{x}$, where $\Omega_{x}$ is the curvature of the QPC introduced above. Additionally, the width of the conductance step, i.e. the gate-voltage interval in which the conductance increases from zero to one, is also set by $\Omega_{x}$. The general shape of the conductance curve for a parabolic barrier in the absence of interactions is a step described by a Fermi-function, as was shown by Büttiker in Ref. 15. If one switches on onsite interactions, the conductance curve becomes asymmetric and flattens increasingly at the top. This effect can be traced back to the fact that when the barrier top drops below the chemical potential as the QPC is being opened up, the maximum in the LDOS just above the barrier top (called van Hove ridge in Ref. [7]) is aligned with the chemical 
potential, thereby strongly enhancing interaction effects. It turns out that the effective onsite interaction strength is in fact given by

$$
U_{j}^{\mathrm{eff}}=U \cdot \mathcal{A}_{j}^{0}(\mu)
$$

where

$$
\mathcal{A}_{j}^{0}(\omega)=-\frac{1}{\pi} \operatorname{Im} \mathcal{G}_{j j}^{0}\left(\omega+i 0^{+}\right)
$$

is the non-interacting local density of states per site. Near the barrier center, the resulting $U^{\text {eff }}$ scales like $U / \sqrt{\Omega_{x} \tau}$.

In the QD case, we can vary the width and depth of the middle well, [c.f. Fig. 6 (d,e) below]. Typically, we want to study the crossover between QPC and QD, thus we start out with a QPC setup and lower the potential of the central region to change the geometry to a $\mathrm{QD}$ model. The characteristic physics of the quantum dot is determined by the structure of the discrete levels of the bound states in the well. This quantization leads to a conductance peak whenever such a level crosses the chemical potential and the dot gets filled by one electron more. In the interacting case, the degenerate levels split on a scale of the interaction strength $U$. However, there is a further effect: the odd valleys, i.e. the regions between the peaks where the dot contains an odd number of electrons, become conductance plateaus with $G_{Q} \approx 1$. This behavior reflects the occurrence of the Kondo [25] effect since the singly-occupied dot level behaves like a localized spin coupled to a fermionic bath.

In this work, we will apply our eCLA first to the same type of onsite models of QPCs as used by BHD [7, 8, 19] and analyse the resulting effects. Importantly, we find that in comparison to the CLA used previously, the eCLA yields an improved stability of the fRG flow in the case of large bare LDOS at the chemical potential. This improvement allows us to additionally study the QPC-QD crossover, which involves a very high LDOS due to the flat barrier top that occurs in this transition. Using the CLA, it had not been possible to study this transition when the barrier top lies close to the chemical potential $\mu$, since the CLA equations did not converge. Due to this problem, in the real-space approach chosen by Heyder et al. [19] it was not possible to study dots which contain just a few electrons. Since our new feedback scheme significantly ameliorates the convergence problem, we are now able to study the crossover from a QPC to a QD which is just occupied by a single electron. This will be shown in section III D.

\section{Increasing the feedback length}

Let us now study the influence of the feedback length $L$ on the zero-temperature linear conductance [26],

$$
g=\frac{1}{2} \sum_{\sigma}\left|2 \pi \rho^{\sigma}\left(\mu+i 0^{+}\right) \mathcal{G}_{-N^{\prime} N^{\prime}}^{\sigma}\left(\mu+i 0^{+}\right)\right|^{2}
$$

Here $\rho(\omega)$ is the density of states at the boundary of a semi-infinite tight-binding chain; two such chains represent the two one-dimensional non-interacting leads, coupled to the central interacting region. Let us first look at the QPC case. We are interested in the shape of the conductance trace as a function of applied gate voltage and how this shape changes with external parameters, such as applied magnetic field.

For pure onsite interactions, it is natural to choose the feedback length $L=0$. This is what has been done in Refs. 7, 8, 19, and 27, and the results have been discussed therein in detail. Here, we will allow a nonzero $L$, although the actual interaction is purely onsite. This implies that a certain class of additional third order terms will be generated during the RG flow which introduce a better coupling between the channels in the sense of the feedback in Eq. (23). For $L \rightarrow N$ the third-order truncated static fRG scheme is recovered fully regarding the spatial structure of the two-particle vertex (but not for its frequency structure, since we are using the static approximation). Figs. 3(a) to (c) show the conductance $G$ as a function of gate voltage $V_{\mathrm{g}}$ for different values of magnetic field $B$, calculated at fixed $U$ and different values of feedback parameters $L$. Increasing the latter from $L=0$ to $L=3$, c.f. Fig. 3(b), leads to quantitative but not qualitative changes in the shape of the conductance curves - the main effect is that the width of the $B$-induced subplateau decreases. In this regard, increasing $L$ has a qualitatively similar effect to decreasing $U$ (at $L=0$ ), c.f. Fig. 3(d) to (f). Note, though, that increasing $L$ hardly affects the $V_{g}$ position of the conductance step, whereas decreasing $U$ does shift the step slightly towards higher $V_{g}$ values, as expected physically due to the lowering of the Hartree barrier. Increasing the feedback beyond $L=5$ does not lead to any significant quantitative changes, as can be seen in Fig. 3(c) where $L=5$ (black line) is directly compared with $L=8$ (red dashed line). Hence, for the present model convergence is reached for $L \lesssim 5$. In general this value depends on the strength of interaction $U$, and more importantly on the actual shape of the barrier.

In Fig. 4 we study the convergence behavior as function of the feedback length $L$ more thoroughly, for four different values of the geometric length scale $l_{x} / a$ [Eq. (41)] which is the width of the region where the LDOS is enhanced. To determine the convergence behavior, we first chose a large value $L_{\text {large }}$ (here $L_{\text {large }}=21$ ) for which $\max _{V_{g}}\left|g_{L_{\text {large }}}\left(V_{g}\right)-g_{L_{\text {large }}-1}\left(V_{g}\right)\right|$ is smaller than $10^{-4}$, i.e for which we can assume that the conductance is converged against its limit. We then plot

$$
\Delta g_{L}:=\max _{V_{g}}\left|g_{L}\left(V_{g}\right)-g_{L_{\text {large }}}\left(V_{g}\right)\right|
$$

as a function of $L$. For our purposes, as for the plots in Fig. 3, we will regard that the conductance as being converged when $\Delta g_{L} \leq 0.5 \cdot 10^{-2}$. In Fig. 4 this criterium is indicated by the dashed line. The inset shows the smallest $L$ (named $L_{C}$ ) for which the conductance is converged as a function of $l_{x} / a$. We see that for all models under our consideration $L_{C}$ is comparable to $l_{x} / a$. Due to this convergence, the number of vertex components can safely 


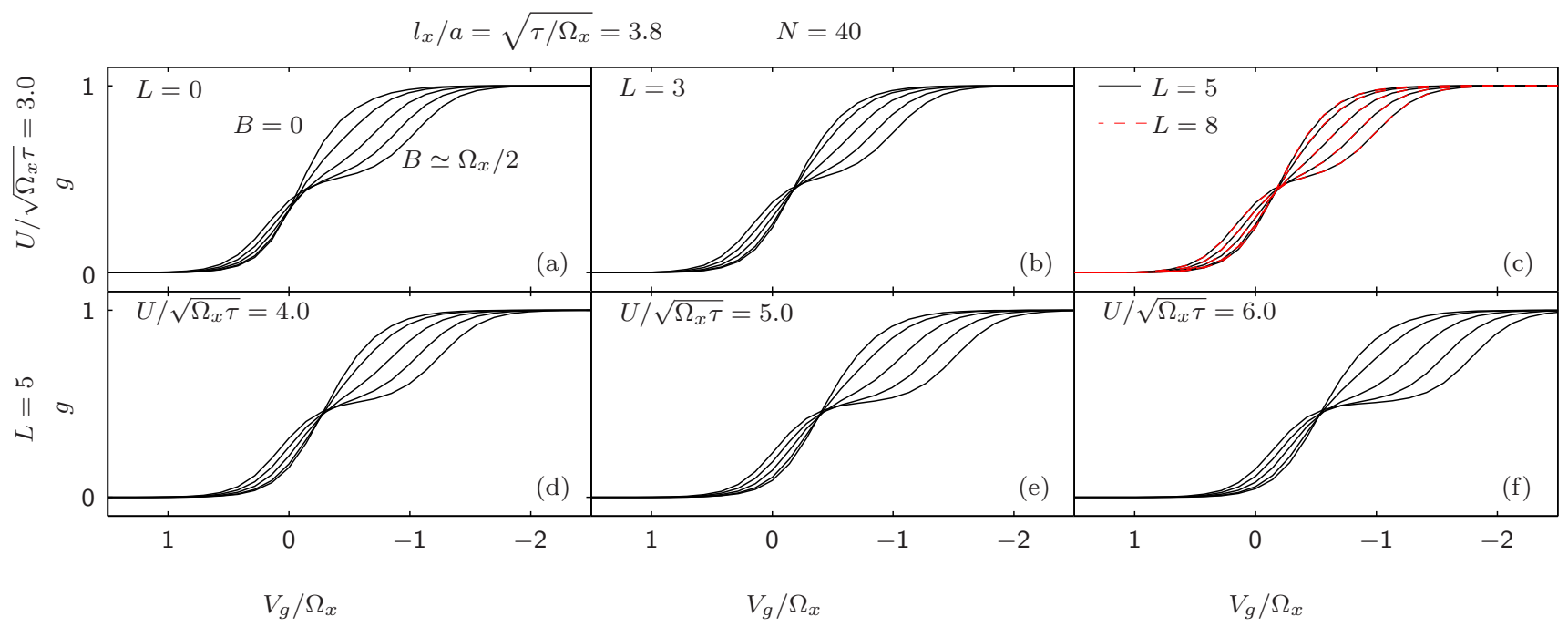

Figure 3. Linear conductance $g$ calculated using the static eCLA for five equidistantly chosen magnetic fields $B$ between 0 and $\Omega_{x} / 2$. (a-c) Conductance at fixed $U / \sqrt{\Omega_{x} \tau}=3.0$, and four values of $L$. (d-f) Conductance at fixed $L=5$, for three values of $U / \sqrt{\Omega_{x} \tau}$.

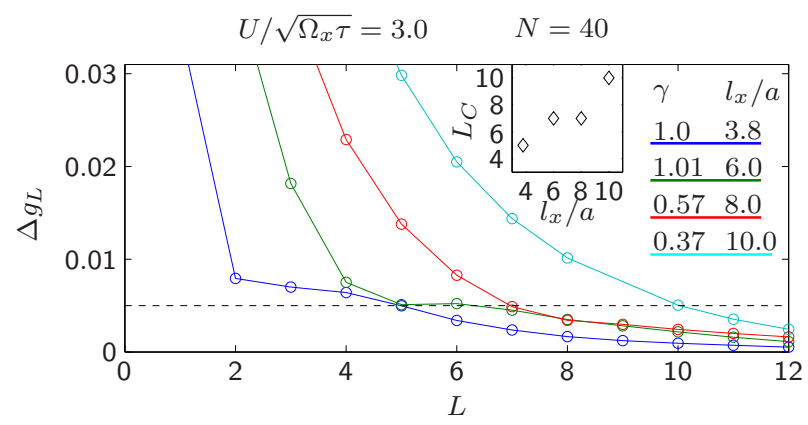

Figure 4. Convergence behavior of the conductance for different values of $l_{x} / a$, where $\Delta g_{L}$ is defined in Eq. (49). The parameters for the $l_{x} / a=3.8$ data are the same as in Fig. 3. For the larger $l_{x}$ values the chemical potential was chosen as $\mu=-1.7$ and the parameter $\gamma$ was varied. The inset shows the dependence of $L_{C}$ on $l_{x}$.

be reduced from $\mathcal{O}\left(N^{4}\right)$ to $\mathcal{O}\left(N^{2} L^{2}\right)$, where $L \approx l_{x} / a$. It would be interesting to investigate if this number can be reduced even further, a next possible candidate being $\mathcal{O}\left(N L^{3}\right)$, by studying the structure of the vertex in more detail. This is, however, beyond the scope of this work and we leave this question for further research.

The extended feedback between the channels becomes increasingly important with increasing interaction strength. For $L=5$ the eCLA yields meaningful, converged results for interaction values for which the $L=0$ flow obtained by CLA is divergent. This is the case for $U \gtrsim 4 \sqrt{\Omega_{x} \tau}$. Figs. 3(d) to (f) show the conductance for such large values of interaction and $L=5$. The qualitative behaviour is unchanged w.r.t. smaller values of the interaction, and the quantitative strength of the impact of the interaction increases continuously, in that the width of the spin-split subplateau increases with $U$.

To shed light on the effect of the enhanced coupling

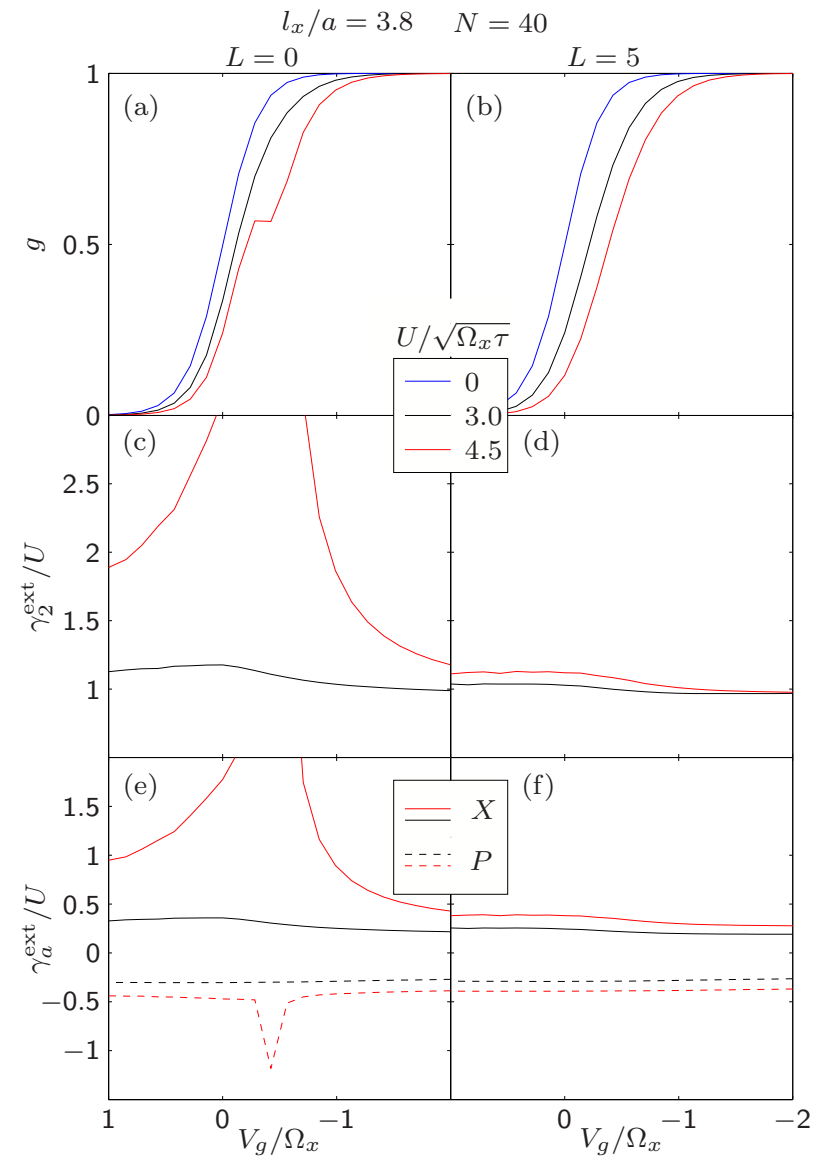

Figure 5. Conductance and vertex quantities calculated for the two feedback lengths $L=0$ (left column) and $L=5$ (right column) with three different effective interaction strengths $U / \sqrt{\Omega_{x} \tau}$, at zero magnetic field. 
between the channels, we now analyse the resulting twoparticle vertex quantitatively, by studying its extremal value

$$
\gamma_{2}^{\text {ext }}=\max _{q_{1}^{\prime} q_{2}^{\prime} q_{1} q_{2}}\left|\gamma_{2}\left(q_{1}^{\prime}, q_{2}^{\prime} ; q_{1}, q_{2}\right)\right|
$$

where the $q$ 's stand here both for site and spin indices. Furthermore, we identify the two most contributing parts to these value as

$$
\begin{aligned}
& \gamma_{x}^{\text {ext }}=\max _{j_{1}^{\prime} j_{2}^{\prime} j_{1} j_{2}} \gamma_{x}\left(j_{1}^{\prime} \uparrow, j_{2}^{\prime} \downarrow ; j_{1} \uparrow, j_{2} \downarrow\right) \\
& \gamma_{p}^{\text {ext }}=\min _{j_{1}^{\prime} j_{2}^{\prime} j_{1} j_{2}} \gamma_{p}\left(j_{1}^{\prime} \uparrow, j_{2}^{\prime} \downarrow ; j_{1} \uparrow, j_{2} \downarrow\right) .
\end{aligned}
$$

Note that we used the minimum in the definition of $\gamma_{p}^{\text {ext }}$, since the $\gamma_{p}$ contribution is mainly negative, whereas $\gamma_{x}$ is dominated by its positive part. Fig. 5 shows these quantities and the conductance as a function of $V_{g}$ for $L=0$ and $L=5$. The main message of this figure is that for intermediate interaction strength (solid black curves) the flow converges for both $L=0$ (left column) and $L=5$ (right column) and yields qualitatively the same results for the conductance in Fig. 5 (a,b). If, however, one increases the interaction strength further (red solid curves) the flow for $L=0$ starts to diverge [Fig. $5(\mathrm{c}, \mathrm{e})]$ and the values of physical observables computed from it become wrong, reflected for example in the kink of the red conductance curve in Fig. 5 (a). A good measure for the behavior of the flow is the maximum value of the two-particle vertex, plotted in Fig. 5 (c,d). We see that the kink in the conductance curve corresponds to a very large value of $\gamma_{2}^{\text {ext }} / U=58.2$ [lying outside of the range of Fig. 5(c)]. In contrast, for $L=5, \gamma_{2}^{\text {ext }}$ as well as the conductance stay well behaved and, in fact, the flow converges without problems [Fig. 5 (b,d)]. In order to shed light on this stabilizing effect of the enhanced feedback, we show in Fig. 5 (e,f) the $P^{\uparrow \downarrow}$ and $X^{\uparrow \downarrow}$ part of the channels, which constitute the contributions to $\gamma_{2}^{\text {ext }}$ with the largest moduli. In the case of intermediate interaction (black curves) the $X$ and $P$ contributions are of the same order of magnitude but differ in their relative sign. If one looks at the completely uncoupled channels, i.e. the pure ladder contributions (c.f. the study in Ref. 8) and increases the interaction strength, the $\mathrm{X}$-channel is the first one to diverge. Our interpretation of the stabilizing effect is now as follows. Since the channels are coupled, a slight increase in the modulus of the X-channel leads via the feedback to a slight increase of the modulus of the P-channel, and due to their relative sign difference they partially cancel, so that the resulting additional contribution to $\gamma_{2}$ is small. If the effective interaction becomes too strong, this ameliorating effect eventually breaks down and the flow diverges. In the $L=5$ case, we take much more feedback between the individual channels into account than for $L=0$ and it is therefore reasonable that the divergence point of the flow is shifted toward larger effective interactions.

\section{Crossover between a closed QPC and a QD}

As we have seen above, the increase of the feedback length $L$ leads to a more stable fRG flow in regions for high LDOS, corresponding to a large effective interaction strength. This stabilization effect enables us to study parameter regimes that have been hard to treat with previous fRG schemes. We illustrate this below for a situation known to suffer from fRG divergence problems, namely the crossover from a QPC to a QD. In Ref. 19 it was found that when using the CLA (called "fRG2" there), the fRG flow for this transition suffers from divergences if the flat barrier top is too close to the chemical potential. For this reason, it was not possible for fRG2 to smoothly describe how the dot filling increases with decreasing $V_{g}$, and the region where no or only a few electrons occupy the dot remained inaccessible within the CLA. The eCLA enables us now to study precisely this interesting region. (In Ref. 19, this regime was treated instead using a simpler fRG scheme without vertex flow ("fRG1"). Although this did qualitatively produce the Kondo physics that is expected if the QD occupancy is odd, Ref. 19 argued that fRG1 is generically less reliable than fRG2. For example, for a QPC geometry, it underestimates the scewing of the zero-temperature conductance step that is characteristic for the 0.7-anomaly. For this reason, the detailed studies of QD-QPC crossovers performed in Ref. 19 were all limited to deep dots, studied using fRG2.)

In Fig. 6 (a) we show the conductance curve for the crossover between a closed QPC and a QD, in which the first two bound state levels cross the chemical potential as the dot is made deeper. This level structure is illustrated in Fig. 6 (b,c) where we show the noninteracting LDOS of the dot structure for the two gate voltages indicated by the black markers in Fig. 6 (a). Both of these gate voltages lie within regions where the sharp LDOS maximum associated with a bound state near $\omega=\mu$ causes convergence problems if the feedback length $L$ is small, but not if $L$ is chosen sufficiently large, which is possible within the eCLA.

When varying gate voltage, we can see Kondo plateaus in the conductance arising in the $V_{g}$ regions where the occupation of the dot is odd. This is illustrated in Fig. 6 (d,e), where we show the site-resolved density, again for the two $V_{g}$ values indicated in (a). We see that the electrons are localized within the QPC, which here had a width of 20 sites. When the densities within the QPC are integrated, we indeed obtain approximately one electron for the first plateau and three electrons for the second plateau.

These Kondo plateaus, caused by Kondo screening of the dot spin, get suppressed with increasing magnetic field since the spin degeneracy is broken. This suppression happens in the first and second Kondo plateau for magnetic fields on the scale $\sim 1 \cdot 10^{-4} \tau$ (solid black lines), and $\sim 3 \cdot 10^{-4} \tau$ (dashed black lines), respectively. A quantitative extraction and analysis of the Kondo scales of the setup is beyond the scope of this paper. Our main purpose here is to illustrate that the finite-ranged feedback of eCLA enables us to treat a parameter regime 

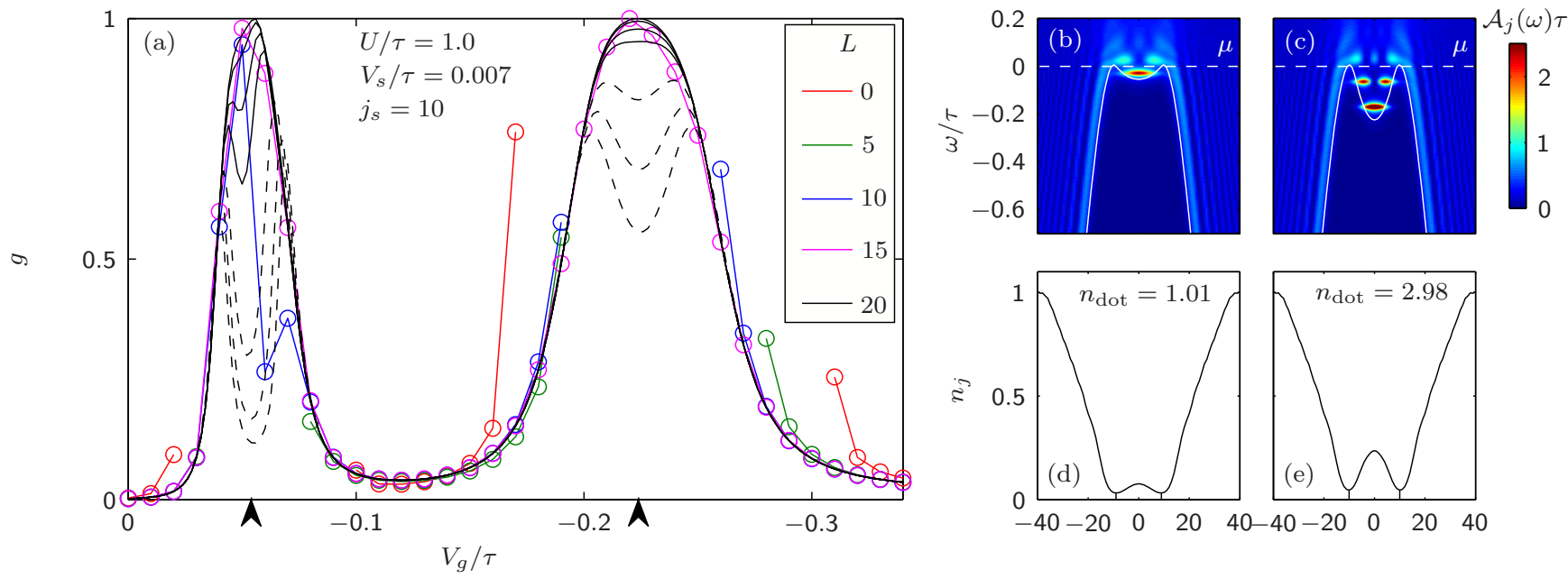

Figure 6. The crossover from a QPC to a QD. (a) The conductance as function of gate-voltage $V_{g}$, calculated for several magnetic fields (black solid lines: $B=0,1,2,3 \cdot 10^{-4}$, black dashed lines: $B=6,9,12 \cdot 10^{-4}$ ) with feedback-length $L=20$. Colored symbols indicate the conductance values obtained with smaller feedback lengths. (b), (c) Non-interacting LDOS (color scale) and barrier shape (solid white curve) for the two gate voltages marked by the left and right vertical arrows in (a), respectively. Horizontal white dashed lines indicate the chemical potential $\mu$. (d), (e) The electron density per site, $n_{j}$, again computed for the two gate voltages indicated in (a). Summing $n_{j}$ over all sites between the two density minima yields $n_{\text {dot }}=1.01$ and $n_{\text {dot }}=2.98$.

which was not accesible with previous fRG schemes and produces qualitatively correct Kondo physics. To outline this, we have indicated in Fig. 6 (a) how the range of convergence increases with increasing $L$ from 0 to 30 . We see that the $L=0$ method is only convergent in the parameter regimes where the occupancy of the dot is even and hence the conductance is small. By increasing $L$ from 0 over 5 to 10 , we see that also the conductance plateaus become more and more visible. At $L=20$ the whole Kondo plateau is accesible. Upon further increasing the feedback up to $L=30$ (not shown here), we find that the conductance results for $L=20$ are already properly converged.

\section{FINITE-RANGED INTERACTIONS}

In this section we consider a model of a QPC with an interaction whose range extends over up to $N$ sites, in contrast to the purely onsite interaction studied in Sec. III. The purpose of this study is to illustrate the potential of the eCLA to deal with finite-ranged interactions in a setting where the screening of a longer-ranged interaction comes into play, and to take a first step towards exploring the physical consequences of screening. We should emphasize, though, that we do not aim here to achieve a fully realistic treatment of screening in a QPC. That would require including higher-lying transport modes (we consider just the lowest-lying one), which would go well beyond the scope of the present paper.
Our model is described by the following Hamiltonian:

$$
\begin{aligned}
\hat{H}= & \sum_{i j \sigma}\left[E_{j}^{\sigma} \hat{n}_{j \sigma}-\tau\left(d_{j \sigma}^{\dagger} d_{j+1 \sigma}+\text { h.c. }\right)\right] \\
& +\frac{1}{2} \sum_{i, j, \sigma, \sigma^{\prime}} U_{i j} \hat{n}_{i \sigma} \hat{n}_{j \sigma^{\prime}}\left(1-\delta_{i j} \delta_{\sigma \sigma^{\prime}}\right) .
\end{aligned}
$$

Here $E_{j}^{\sigma}$ is chosen as described in section III, and $U_{i j}$ can differ from zero for all sites with separation $|i-j|<L_{U}$, where $L_{U}$ determines the bare interaction range. Note that we now also have a bare interaction between electrons with the same spin, which was absent in the onsite case. In the previous section, the interaction strength was controlled by a single value $U$ [c.f. eq. (45)] and treated as a tunable parameter, whose strength was varied by hand. However, now $U_{i j}$ is a matrix with $N^{2}$ parameters, and we need to specify its form explicitly. For this we start with a continuous $3 \mathrm{D}$ model of a QPC, and for the Hilbert spaces associated with transverse motion in the $y$ - and $z$ directions we reduce the dimensionality down to one, by taking into account only the ground states of the respective confining potentials, c.f. Ref. 28. In this way, we arrive at a continuous effective theory in 1D for the $x$ direction, which in a last step is discretized using a finite difference method, already applied by BHD in Ref. 8. We use the resulting model to compute the conductance and the density profile of a QPC, and study their dependence on the screening effects of the long-ranged interaction and the geometric dimensions of the QPC. 


\section{A. Derivation of a 1-dimensional Hamiltonian}

We start from the Hamiltonian $\hat{H}=\hat{H}_{0}+\hat{H}_{1}$ with

$$
\begin{aligned}
\hat{H}_{0}= & \sum_{\sigma} \int d^{3} r \hat{\Psi}_{\sigma}^{\dagger}(\mathbf{r})\left(V_{\mathrm{QPC}}(\mathbf{r})-\frac{\hbar^{2}}{2 m} \nabla^{2}\right) \hat{\Psi}_{\sigma}(\mathbf{r}) \\
\hat{H}_{1}= & \frac{1}{2} \sum_{\sigma_{1}, \sigma_{2}} \int d^{3} r_{1} \int d^{3} r_{2} U\left(\mathbf{r}_{1}-\mathbf{r}_{2}\right) \\
& \times \hat{\Psi}_{\sigma_{1}}^{\dagger}\left(\mathbf{r}_{1}\right) \hat{\Psi}_{\sigma_{2}}^{\dagger}\left(\mathbf{r}_{2}\right) \hat{\Psi}_{\sigma_{2}}\left(\mathbf{r}_{2}\right) \hat{\Psi}_{\sigma_{1}}\left(\mathbf{r}_{1}\right),
\end{aligned}
$$

where the fermionic field $\hat{\Psi}_{\sigma}^{\dagger}(\mathbf{r})$ creates an electron with spin $\sigma$ at the continuous position variable $\mathbf{r}$. The interaction is of screened Coulomb form with screening length $l_{s}$ and relative dielectric constant $\kappa$, which is given in ESU-CGS units by:

$$
U\left(\mathbf{r}_{1}-\mathbf{r}_{2}\right)=\frac{e^{2}}{\kappa}\left(\frac{1}{\left|\mathbf{r}_{1}-\mathbf{r}_{2}\right|}-\frac{1}{\sqrt{\left|\mathbf{r}_{1}-\mathbf{r}_{2}\right|^{2}+l_{s}^{2}}}\right),
$$

c.f. Hirose et al. [29]. This interaction form results from taking image charges on the top gate into account, which is positioned at a distance of $l_{s} / 2$ above the 2DEG. We use a QPC potential given by

$$
V_{\mathrm{QPC}}(x, y, z)=\left[\alpha V(x)+m^{*} \frac{\Omega_{y}(x)^{2}}{\hbar^{2}} \frac{y^{2}}{2}\right] \Delta(z),
$$

with $\Omega_{y}(x)=2 \beta V(x)$, and $m^{*}=0.067 m_{e}$ is the effective mass of GaAs. The function $\Delta(z)$ ensures the confinement to the 2DEG and the one-dimensional potential $V(x)$ which enters here is the same as that used in our onsite-model studies, Eq. (38). The QPC potential $V_{\mathrm{QPC}}$ has a saddle-like form: it defines a quadratic confinement in $y$-direction with a positiv curvature $\Omega_{y}(x)$ that decreases with increasing $|x|$, whereas the curvature in $x$-direction is negative, with magnitude $\Omega_{x}$. The confinement in $y$-direction disappears for $|x| \rightarrow \infty$, where $V(x)=0$. For the coefficients $\alpha$ and $\beta$, we impose the condition $\alpha+\beta=1$, which turns out to ensure that the effective one-dimensional potential resulting from eliminating the $y$ - and $z$-direction is precisely $V(x)$. We specify the transverse curvature at the center of the QPC to be $\Omega_{y}=\Omega_{y}(0)$, thereby fixing the parameter $\beta=\frac{\Omega_{y}}{2 V(0)}$.

We now project onto the ground state subspace for the transverse directions. With this step, taken for the sake of simplicity, we ignore all transport modes except the one contributing to the first conductance step. For a truly realistic description of screening, the higher-lying modes would have to be taken into account, too. This would lead to stronger screening and an effective interaction of shorter range than that obtained below.

Concretely, we thus represent our quantized fields as

$$
\hat{\Psi}_{\sigma}(\mathbf{r})=\phi_{x}(y) \varphi(z) \hat{\psi}_{\sigma}(x) .
$$

Here $\phi_{x}(y)$ and $\varphi(z)$ are the normalized ground state wave-functions of the confining potentials in the $y$ and $z$ directions, respectively,

$$
\varphi(z)=\sqrt{\tilde{\delta}(z)}
$$

$$
\phi_{x}(y)=\frac{1}{(2 \pi)^{1 / 4} \sqrt{l_{y}(x)}} e^{-y^{2} /\left(4 l_{y}^{2}(x)\right)}
$$

and the operator $\hat{\psi}_{\sigma}(x)$ creates an electron in a state with wavefunction $\delta(x) \phi_{x}(y) \varphi(z)$. In our 2DEG setup, $\tilde{\delta}(z)$ is a peak of weight one, very narrow compared to the scales in $x$ - and $y$-direction, whereas $\phi_{x}(y)$ is the ground state of a harmonic oscillator with characteristic length

$$
l_{y}(x)=\frac{\hbar}{\sqrt{2 m^{*} \Omega_{y}(x)}} .
$$

With this, we arrive at an effective 1D continuous theory described by the effective 1D Hamiltonian

$$
\begin{aligned}
& \hat{H}_{\mathrm{eff}}=\sum_{\sigma} \int d x \hat{\psi}_{\sigma}^{\dagger}(x)\left[\frac{\hbar}{2 m} \partial_{x}^{2}+(\alpha+\beta) V(x)\right] \hat{\psi}_{\sigma}(x) \\
& +\sum_{\sigma_{1}, \sigma_{2}} \int d x_{1} d x_{2} \frac{U\left(x_{1}, x_{2}\right)}{2} \hat{\psi}_{\sigma_{1}}^{\dagger}\left(x_{1}\right) \hat{\psi}_{\sigma_{2}}^{\dagger}\left(x_{2}\right) \hat{\psi}_{\sigma_{2}}\left(x_{2}\right) \hat{\psi}_{\sigma_{1}}\left(x_{1}\right) .
\end{aligned}
$$

We now choose $\alpha+\beta=1$ as stated above, thus ensuring that the resulting effective one-dimensional potential is indeed given by $V(x)$. The matrix elements of the interaction are given by

$$
\begin{aligned}
& U\left(x_{1}, x_{2}\right)=\frac{e^{2}}{\kappa} \sqrt{\frac{1}{2 \pi\left(l_{y}^{2}\left(x_{1}\right)+l_{y}^{2}\left(x_{2}\right)\right)}} \\
& \times\left[\exp \left(\frac{\left(x_{1}-x_{2}\right)^{2}}{4\left(l_{y}^{2}\left(x_{1}\right)+l_{y}^{2}\left(x_{2}\right)\right)}\right) \cdot K_{0}\left(\frac{\left(x_{1}-x_{2}\right)^{2}}{4\left(l_{y}^{2}\left(x_{1}\right)+l_{y}^{2}\left(x_{2}\right)\right)}\right)\right. \\
& \left.-\exp \left(\frac{\left(x_{1}-x_{2}\right)^{2}+l_{s}^{2}}{4\left(l_{y}^{2}\left(x_{1}\right)+l_{y}^{2}\left(x_{2}\right)\right)}\right) \cdot K_{0}\left(\frac{\left(x_{1}-x_{2}\right)^{2}+l_{s}^{2}}{4\left(l_{y}^{2}\left(x_{1}\right)+l_{y}^{2}\left(x_{2}\right)\right)}\right)\right] .
\end{aligned}
$$

For a typical 2DEG of GaAs-AlGaAs the relative dielectric constant has the value $\kappa \approx 12.9 . K_{0}$ is the modified Bessel function of second kind in zeroth order. It diverges logarithmically when its argument approaches zero.

In order to discretize our $1 \mathrm{D}$ continuous theory along the $x$-direction, we set $x:=a \cdot j$ and replace the continuous field $\hat{\psi}_{\sigma}(x)$ by the discrete set of operators $d_{j \sigma}$, where $a$ is the lattice spacing and $j$ the site index. This results in a Hamiltonian of the form (52). Treating the second derivative in the kinetic term using a finite difference method, the single-particle part of the Hamiltonian takes the form $H_{0}=\sum_{i j \sigma} h_{i j}^{\sigma}$, with

$$
h_{i j}^{\sigma}=\left(V_{i}-\frac{\sigma B}{2}\right) \delta_{i j}-\tau\left(\delta_{i, i+1}+\delta_{i, i-1}\right),
$$

where $V_{i}$ is just the discretized version of the effective 1D potential, $B$ is the magnetic field, and $\tau=\frac{\hbar^{2}}{2 m^{*} a^{2}}$ is the hopping matrix element. We define a discretized form of the interaction by

$$
\begin{aligned}
U_{i j} & :=U(a i, a j), \text { if } i \neq j \\
U_{i i} & :=\frac{1}{a^{2}} \int_{a(i-1 / 2)}^{a(i+1 / 2)} d x_{1} \int_{a(i-1 / 2)}^{a(i+1 / 2)} d x_{2} U\left(x_{1}, x_{2}\right),
\end{aligned}
$$


where we treat the on-site case separately, since $U\left(x_{1}, x_{2}\right)$ has an integrable singularity as $x_{1}$ approaches $x_{2}$. The above treatment presupposes that the transverse wavefunctions do not change significantly on a scale set by $a$. If $a$ is much smaller than the characteristic length of the electrostatic potential, the above discretization scheme correctly captures the physical behavior of the continuous theory while regularizing the short-distance of the interaction, with $U_{i i}=-\frac{e^{2}}{\kappa \sqrt{\pi} l_{y}(a i)} \cdot \log \left[a / l_{y}(a i)\right]+\mathcal{O}(1)$ for $a \rightarrow 0$.

Having arrived at the discretized Hamiltonian (52), let us take a final look at the parameters that characterize our system. From the dimensionful constants $\hbar, e^{2} / \kappa$ and $m^{*}$ one can construct an intrinsic length scale $\left[\frac{\hbar^{2}}{m^{*} e^{2}} \kappa\right] \approx$ $10 \mathrm{~nm}$ and intrinsic energy scale $\left[\frac{m^{*} e^{4}}{2 \hbar^{2} \kappa^{2}}\right] \approx 5.5 \mathrm{meV}$. It is possible to express all our model's length and energy scales in terms of these two dimensionful constants. However, it is often convenient to be able to relate quantities like the gate-voltage dependence of the conductance or the spatial resolution of the density directly to the geometry of the QPC. For this reason, we introduce in our studies below for each QPC a characteristic energy scale $\bar{\Omega}_{x}$, and a corresponding length scale $\bar{l}_{x}=\hbar / \sqrt{2 m^{*} \bar{\Omega}_{x}}$, which we measure in absolute units and which characterize the mean geometry of the QPC barrier. Concretely, we will take for $\bar{\Omega}_{x}$ the curvature of the bare barrier at the renormalized conductance pinchoff gate voltage $V_{g}^{\text {po }}$, where the conductance just begins to increase from zero (and the barrier height is $\varepsilon_{F}+V_{g}^{\mathrm{po}}$ ). All the other geometric quantities are then specified relative to $\bar{\Omega}_{x}$. To be specific, we will characterize our QPC by the following rescaled dimensionless quantities (denoted by tildes):
(i) $\widetilde{\Omega}_{x}=\frac{\bar{\Omega}_{x}}{\mathrm{meV}}$,
(ii) $\tilde{V}_{g}=\frac{V_{g}}{\bar{\Omega}_{x}}$,
(iii) $\widetilde{\Omega}_{y}=\frac{\Omega_{y}(0)}{\bar{\Omega}_{x}}$,
(iv) $\tilde{l}_{s}=\frac{l_{s}}{\bar{l}_{x}}$,
(v) $\widetilde{L}_{\mathrm{bar}}=\frac{L_{\mathrm{bar}}}{\bar{l}_{x}}$,
(vi) $\widetilde{x}=\frac{x}{\bar{l}_{x}}$
(vii) $\widetilde{\Omega}_{y}^{\prime \prime}=\frac{\bar{l}_{x}^{2}}{\bar{\Omega}_{x}}\left[\frac{\partial}{\partial_{x}^{2}} \Omega_{y}(x)\right]_{x=0}$.

$\widetilde{\Omega}_{x}$ describes the longitudinal barrier curvature in units of meV, $\widetilde{V}_{g}$ the normalized gate voltage, $\widetilde{\Omega}_{y}$ the transverse curvature at the barrier center, $\widetilde{l}_{s}$ the screening length, $\widetilde{L}_{\text {bar }}$ the total barrier length which controls the behavior of the flanks, $\widetilde{x}$ the longitudinal coordinate, and $\widetilde{\Omega}_{y}^{\prime \prime}$ the $x$-dependence of the transverse curvature at the barrier center. Note that if one chooses to specify $\widetilde{\Omega}_{x}, \widetilde{\Omega}_{y}, \widetilde{\Omega}_{y}^{\prime \prime}$, $\widetilde{l}_{s}$, and $\widetilde{L}_{\text {bar }}$, this implicitly also fixes $\varepsilon_{F}$ : its values has to be chosen in such a way that the resulting curvature at pinchoff has the specified value $\widetilde{\Omega}_{x}$.

It is instructive to express the interaction $U\left(x_{1}, x_{2}\right)$ of Eq. (61) in terms of the rescaled dimensionless parameters. If we define $U_{b}=e^{2} /\left(\kappa \bar{l}_{x}\right)$, the dimensionless ratio $\tilde{U}\left(\widetilde{x}_{1}, \widetilde{x}_{2}\right)=U\left(x_{1}, x_{2}\right) / U_{b}$ depends only on the dimensionless parameters (65)(ii)-(vii), but not on $\bar{\Omega}_{x}$. Thus, the dependence of the interaction strength (in absolute units) on the longitudinal curvature $\bar{\Omega}_{x}$ of the QPC is

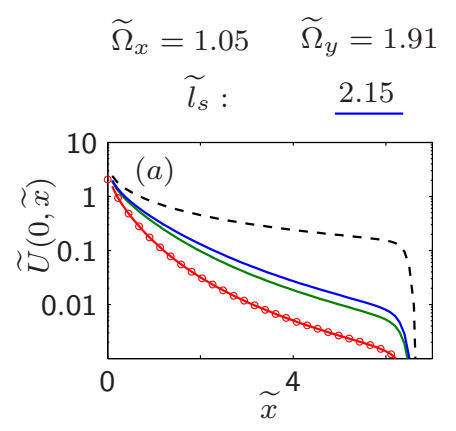

$$
\begin{aligned}
& \widetilde{L}_{\text {bar }}=6.79 \quad \widetilde{\Omega}_{y}^{\prime \prime}=-0.060 \\
& \underline{1.72} \quad \underline{0.86}
\end{aligned}
$$

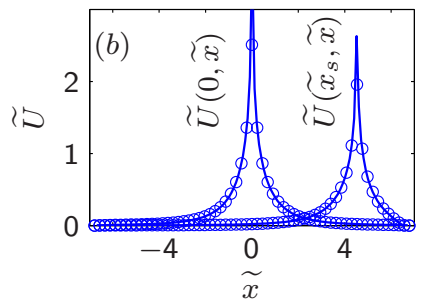

Figure 7. (a) Distance dependence of the bare interaction $\widetilde{U}(0, \widetilde{x})$ between an electron located at the QPC center and one at $\widetilde{x}$, plotted on a logarithmic scale, for three values of $\widetilde{l}_{s}$. The dashed black line shows the limit of $\widetilde{l}_{s} \rightarrow \infty$ and the dots on the lowest curve (red) illustrate the chosen discretization points for the case $N=61$. (b) $\widetilde{U}(0, \widetilde{x})$ (central peak) and $\widetilde{U}\left(\widetilde{x}_{s}=4.5, \widetilde{x}\right)$ (side peak), plotted for $\widetilde{l}_{s}=2.15$ on a linear scale for both negative and positive $\widetilde{x}$ values.

fully encapsulated in $U_{b}$. The corresponding dimensionless parameter

$$
\widetilde{U}_{b}=U_{b} / \bar{\Omega}_{x}=\frac{\sqrt{2 m^{*}} e^{2}}{\kappa \hbar} \frac{1}{\sqrt{\bar{\Omega}_{x}}}
$$

characterizes the effective onsite interaction strength at the barrier center for the present long-ranged interaction model, and plays a role analogous to the parameter $U_{0}^{\text {eff }}=U \cdot \mathcal{A}_{0}^{0}(\mu)$ of Eq. (46) (which likewise scales as $\left.1 / \sqrt{\bar{\Omega}_{x}}\right)$ for the onsite interaction model of section III. Evidently, $\widetilde{U}_{b}$ increases with decreasing $\bar{\Omega}_{x}$, implying that interactions become ever more important the smaller the curvature of the barrier top. Typical values for $\widetilde{U}_{b}$ for the plots below range between 4.2 and 4.9 .

The spatial structure of the long-ranged interaction for typical choices for the physical parameters is shown in Fig. 7. In Fig.7(a), we plotted the dimensionless ratio $\widetilde{U}(0, \tilde{x})=U\left(0, \widetilde{x} \cdot \bar{l}_{x}\right) / U_{b}$ for three values of the rescaled screening length $\widetilde{l}_{s}$, as a function of positive $\widetilde{x}=x / \bar{l}_{x}$. This ratio is independent of $\bar{\Omega}_{x}$ itself, but increases significantly with increasing screening length. In (b), we again show $\tilde{U}(0, \widetilde{x})$ (central peak) and for comparison also $\tilde{U}\left(\widetilde{x}_{s}, \widetilde{x}\right)=U\left(\widetilde{x}_{s} \cdot \bar{l}_{x}, \widetilde{x} \cdot \bar{l}_{x}\right) / \tilde{U}_{b}$ for fixed $\widetilde{x}_{s}=4.5$ as function of $\widetilde{x}$, where the $\widetilde{x}$ range contains now the whole QPC. Due to the reflection symmetry of our system about the QPC center, $\widetilde{U}(0, \widetilde{x})$ is a symmetric function of $\widetilde{x}$. In contrast, $\widetilde{U}\left(\widetilde{x}_{s}, \widetilde{x}\right)$ is an asymmetric function of $\widetilde{x}$ around the point $\widetilde{x}=\widetilde{x}_{s}$, decreasing more quickly when $\widetilde{x}-\widetilde{x}_{s}$ becomes large positive than large negative, because the transverse potential is wider in the former case. This widening of the transverse potential is also the reason why $\tilde{U}\left(\widetilde{x}_{s}, \widetilde{x}\right)$ as a function of $\widetilde{x}_{s}-\widetilde{x}$ with fixed $\widetilde{x}_{s}$ is in general smaller than $\tilde{U}(0, \tilde{x})$ as a function of $\widetilde{x}$. 


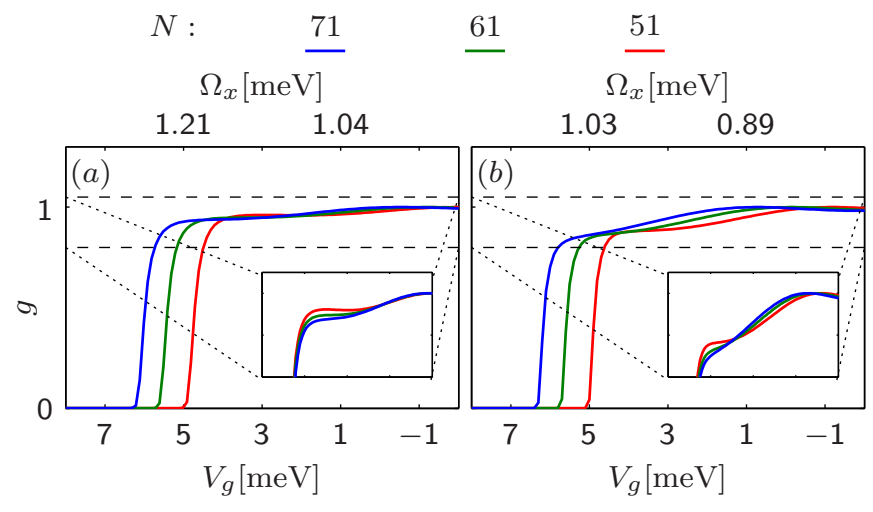

Figure 8. QPC conductance step shape for three choices of the number of discretization points $N$ (with maximal feedback length $L=N-1$ ), for two QPCs with different curvatures. We used the following parameters, in absolut units [c.f. Eqs. (38) and (61)]: In (a), $\gamma=0.85, \varepsilon_{F}=13.89 \mathrm{meV}$, $\Omega_{y}=2.35 \mathrm{meV}, L_{\mathrm{bar}}=146.11 \mathrm{~nm}, l_{s}=46.17 \mathrm{~nm}$; and in (b), $\gamma=0.85, \varepsilon_{F}=11.00 \mathrm{meV}, \Omega_{y}=2.00 \mathrm{meV}, L_{\mathrm{bar}}=158.24 \mathrm{~nm}$, $l_{s}=50.00 \mathrm{~nm}$. The insets zoom into the range $g \in[0.8,1.05]$ and plot $g$ as function of $V_{g}-V_{g}^{\text {po }}$ to align the pinchoffs. When expressed in terms of the dimensionless parameters of Eq. (65), the parameter choices in (a) and (b) differ only in $\widetilde{\Omega}_{x}$. For example, for the middle $N=61$ curves (green) we obtain for panel (a) $\mathfrak{A}=\left\{\widetilde{\Omega}_{x}=1.23, \widetilde{\Omega}_{y}=1.91, \widetilde{L}_{\text {bar }}=\right.$ $\left.6.79, \widetilde{\Omega}_{y}^{\prime \prime}=-0.060, \widetilde{l}_{s}=2.15\right\}$, and for panel $(\mathrm{b}), \mathfrak{B}=\left\{\widetilde{\Omega}_{x}=\right.$ $\left.1.05, \widetilde{\Omega}_{y}=1.91, \widetilde{L}_{\mathrm{bar}}=6.79, \widetilde{\Omega}_{y}^{\prime \prime}=-0.060, \widetilde{l}_{s}=2.15\right\}$.

\section{B. Discretization dependence}

We begin our treatment of long-ranged interactions by investigating to what extent our results depend on the number of discretization points, $N$, with all other parameters held fixed. Fig. 8 shows this dependence for two QPCs whose parameters were chosen to yield somewhat different ranges of $\Omega_{x}$ curvatures. The first point to notice involves the $V_{g}$ value of the conductance pinchoff: whereas in the absence of interactions it occurs near $V_{g}=0$, turning on our long-ranged interactions shifts it towards the left, i.e. towards a larger gate voltage. This behavior is unphysical, since for any fixed $V_{g}$ at which the density is nonzero, turning on interactions should generate a Hartree barrier that causes the conductance to decrease, not increase. We suspect that this unphysical behavior is a fRG artefact, possibly due to our use of the static approximation. We leave the issue of exploring what will happen when using a dynamic version of our eCLA as a topic for future study. We remark, however, that similar unphysical shift artifacts where encountered in Ref. 8 when comparing various different fRG methods that treated the details of the vertex flow in somewhat different ways. Nevertheless, although the $V_{g}^{\text {po }}$ values of the conductance curves in Ref. 8 depended on methological details, the overall shape of the conductance steps were essentially the same, i.e. when plotted as functions of $V_{g}-V_{g}^{\text {po }}$, they coincided. We find a similar trend here: if we increase $N, V_{g}^{\text {po }}$ increases, because changing $N$ slightly changes the strength and shape of the interaction function $U_{i j}$, causing corresponding changes in $V_{g}^{\text {po }}$ and $\bar{\Omega}_{x}$; however, the shape of the conductance steps in Figs. 8 (a),(b) seems at least qualitatively convergent when $N$ increases [c.f. insets in (a) and (b)], despite the $N$ dependence of the step's position. For the remainder of this paper we will therefore only address the overall shape of the conductance step.

In Fig. 8(a),(b) we expressed all parameters in terms of absolute units. In most of the remaining plots where physical properties are discussed, we use instead the more convenient dimensionless quantities introduced in Eq. (65) (and denoted by tildes). We have also extracted these dimensionless parameters for Figs. 8(a),(b) and summarized them for further use in the parameter sets $\mathfrak{A}$ and $\mathfrak{B}$ given in the caption of Fig. 8 .

In Fig. 8 we used the maximal feedback length $L=$ $N-1$ to fully take interactions over the whole QPC into account. However, due to numerical costs, this limited the number of sites that could be treated to $N \leq 71$. For this reason, we have also explored using a cutoff length $L_{U}$ for the interaction range, setting $U_{i j}=0$ for $|i-j|>$ $L_{U}$. The resulting conductance curves for different $L_{U}$ are shown in Fig. 9. We first note that when the cutoff length $L_{U}$ becomes smaller than the characteristic length $l_{x} / a \approx 4.4$ of the QPC, we recover the conductance shape for short-ranged interactions. This behavior is analogous to that obtained in Fig. 10 below, when reducing the screening length $l_{s}$ below $l_{x}$. Furthermore, we find rapid convergence when increasing $L$ beyond $L_{U}$ for a fixed $N$ : for example, Fig. 9 contains two curves for $L_{U}=$ 10 , one computed with $L=60$ (solid), the other with $L=15$ (dashed), which essentially coincide. However, the shape of the conductance step becomes independent of $L_{U}$ only for rather large values of $L_{U}$, implying that the tail of the long-ranged interaction actually matters significantly. Therefore, we did not pursue using $L_{U}<N$ any further and for the remainder of this work show only data obtained without interaction cutoff and with full feedback length, $L=N-1$.

\section{Effects of long-ranged interactions on QPC properties}

After these technical considerations, let us now study how the fact that the interaction range is not zero affects the QPC properties. For this, we first briefly discuss the dependence of our finite-ranged interaction on the given physical parameters and then study the resulting consequences on the conductance and the density. As pointed out earlier, this study does not aim to achieve a fully realistic description of screening in a QPC, but rather serves as a first illustration of the potential of the eCLA for treating a model with reasonably long-ranged interactions.

Figs. 10(a) and (b) show, for two different values of the curvature $\widetilde{\Omega}_{x}$, respectively, three conductance curves corresponding to the three choices of $\widetilde{l}_{s}$ used in Fig. 7 (a). For both choices of $\widetilde{\Omega}_{x}$, we obtain an onsite-like conductance step shape when $\widetilde{l}_{s}$ is small. When $\widetilde{l}_{s}$ is increased, i.e. 


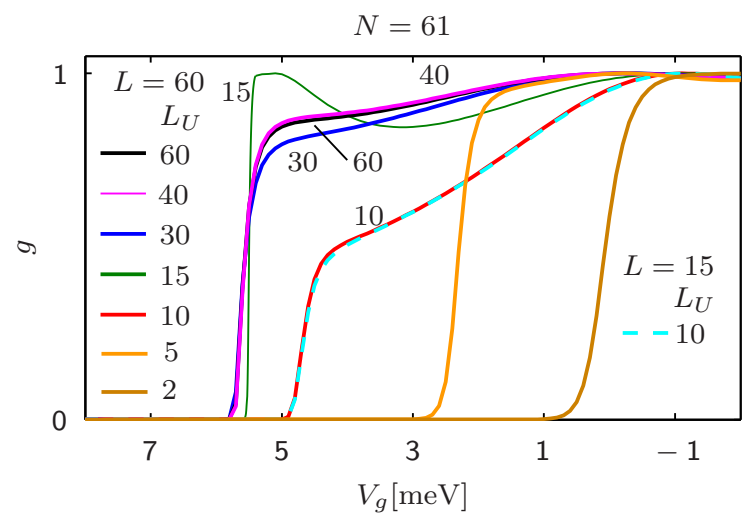

Figure 9. QPC conductance curves at fixed $N$, calculated with feedback length $L=N-1$ for several values of the interaction cutoff $L_{U}$ (solid lines), and with $L=15$ for $L_{U}=$ 10 (dashed line). The QPC parameters were chosen as in Fig. 8(b). Note that while convergence in $L$ is rapid, the conductance becomes independent of the cutoff length only for $L_{U}>40$. Furthermore, for $L_{U} \lesssim l_{x} / a \approx 4.4$ we recover the conductance shape of short-ranged interactions.
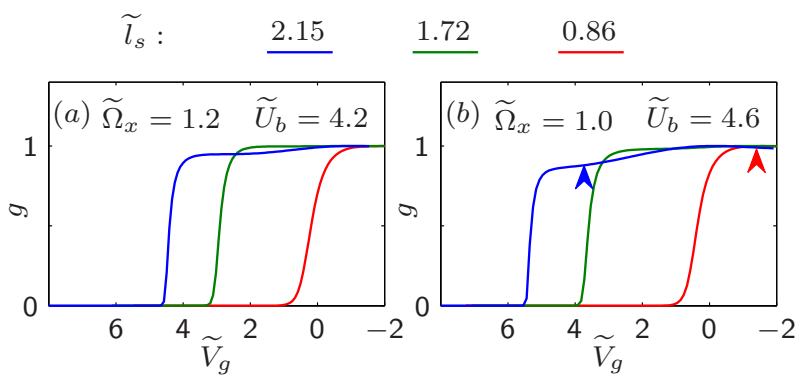

Figure 10. (a), (b) The conductance curves corresponding to the interactions depicted in Fig. 7 (a), for two different QPC mean curvatures $\widetilde{\Omega}_{x}=1.2$ and $\widetilde{\Omega}_{x}=1.0$, respectively. The arrows at the right (red) $\widetilde{l}_{s}=0.86$ and the left (blue) $\tilde{l}_{s}=2.15$ curve in (b) indicate the gate voltages $\widetilde{V}_{g}=-1.43$ and $\widetilde{V}_{g}=3.73$ at which the density profiles in Figs. 11(a) and (b) were calculated, respectively.

when the amount of screening is reduced, the step shape acquires some additional features, such as the emergence of a "preplateau" at a value of $g$ slightly lower than 1 , followed by a much slower increase towards 1 in Fig. 10(a). These features are more pronounced for the longer QPC (i.e. smaller curvature) of Fig. 10(b), where the conductance quickly reaches a preplateau around $g \simeq 0.8$ and thereafter increases much more slowly.

In order to explore the origin of this behavior, we show in Fig. 11(a) and 11(b) two density profiles (thin lines), calculated, respectively, for two fixed parameter choices from Fig. 10(b), indicated in the latter by the right (red) marker for $\widetilde{l}_{s}=0.86, \widetilde{V}_{g}=-1.43$ and the left (blue) marker for $\widetilde{l}_{s}=2.15, \widetilde{V}_{g}=3.73$. In Fig. $11(\mathrm{~b})$, for which the rescaled screening length $\widetilde{l}_{s}$ is larger, we observe three qualitative changes relative to Fig. 11(a). First, the flanks of the density profile are somewhat steeper. Sec-

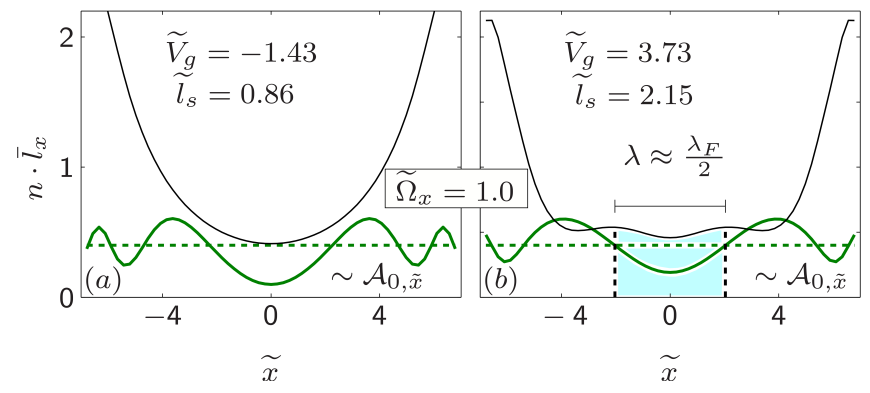

Figure 11. Density profiles (thin lines) calculated for two fixed parameter choices from Fig. 10, indicated for panels (a) and (b) by the right and left arrows in Fig. 10(b), respectively. For comparison, the thick lines depict (a vertically rescaled version of) the imaginary part of the interacting single-particle propagator at the chemical potential, $\mathcal{A}_{0, \widetilde{x}}=-\frac{1}{\pi \bar{l}_{x}} \operatorname{Im} G_{0, \widetilde{x}}^{R}(\omega=0)$. Horizontal dashed lines indicate where $\mathcal{A}_{0, \widetilde{x}}=0$. In (b), the distance between the two density maxima (marked by the dashed vertical lines) is $\lambda=3.62 \bar{l}_{x}$. This agrees well with two estimates of $\lambda_{F} / 2$, either from the distance between the two central zeros of $\mathcal{A}_{0, \widetilde{x}}$ finding $\lambda_{F} / 2=3.82 \bar{l}_{x}$ or from the mean density $\bar{n}$ in the center of the QPC (shaded region) finding $\lambda_{F} / 2=3.55 \bar{l}_{x}$.

ond, the spatial region in which the density is low has become wider. And third, in this low-density region the density shows some weak density oscillations that are absent in Fig. 11(a).

The first two features suggest that the long-range interactions have generated a renormalized barrier whose shape has a flatter top and steeper flanks than the bare parabolic barrier. This flattening occurs because the bare density is larger in the flanks than near the center, hence the upward Hartree-type shift of the barrier potential, which is proportional to the bare density, is larger in the flanks than near the center. The upward renormalization in the flanks becomes stronger the larger the interaction range, because then the upward Hartree-type shift at a given site is determined by a weighted average of the density over a range of nearby sites (whose extent is set by the screening length), and since the bare density profile is convex, the sites in the flanks contribute more strongly.

To shed further light on the third feature, namely the weak density oscillations in the low-density region, we compare their oscillation period with estimates for the "local Fermi wavelength" $\lambda_{F}$ at the QPC center, which can be extracted from either the interacting Green's function or the mean density in the center of the QPC. To illustrate the first method, the thick lines in Figs. 11(a) and (b) indicate the oscillatory behavior of $\mathcal{A}_{0, \widetilde{x}}=$ $-\frac{1}{\pi \bar{l}_{x}} \operatorname{Im} G_{0, \widetilde{x}}^{R}(\omega=0)$. For a homogeneous system the Green's function oscillates with period $\lambda_{F}$, and likewise we can here define an effective $\lambda_{F} / 2$ in the middle of the QPC by taking the distance between the two central zeros of the thick line. For Fig. 11(b), the position of these zeros is in good agreement with the position of the density maxima of the QPC (indicated by the two dashed vertical lines), whereas the density in Fig. 11(a) shows no features on the scale of $\lambda_{F}$. An alternative way to extract an effective $\lambda_{F}$ is to calculate the mean density $\bar{n}$ in 


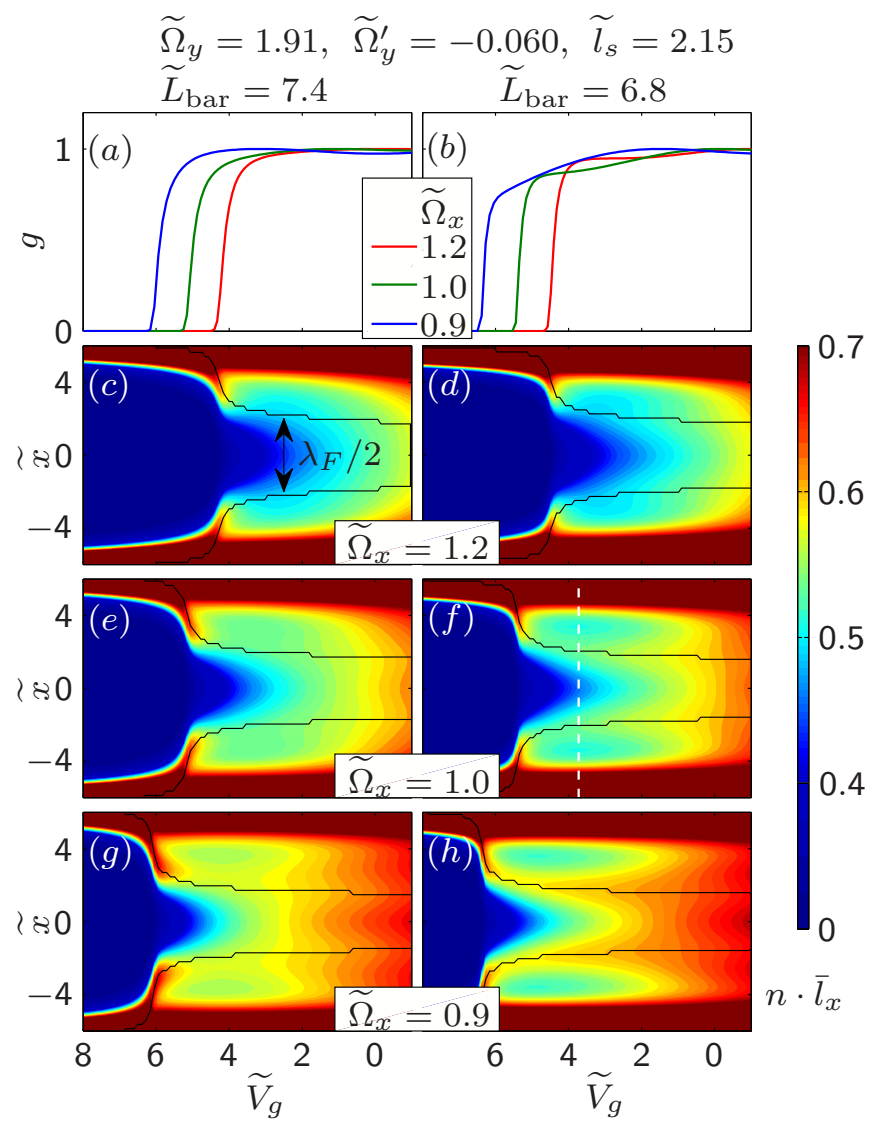

Figure 12. Study of two QPCs with different $\widetilde{L}_{\text {bar }}$, for three choices of $\widetilde{\Omega}_{x}$. The other dimensionless parameters were chosen the same as in $\mathfrak{B}$ [c.f. caption of Fig. 8]. (a,b) Conductance as function of gate voltage, and (c-h) density as function of position and gate voltage. While the conductance changes its shape for both QPCs, the shorter one (b) shows stronger features, preeminently a shoulder in the conductance step. In the density, both QPCs show the development of oscillations with approximate wavelength $\lambda_{F} / 2$, which is determined by the Green's function as in Fig. 11 and indicated by the distance between the black lines. In the last plots $(\mathrm{g})$ and $(\mathrm{h})$ the density oscillations transition at smaller gate voltages from two to three maxima. The cut along the dashed white line in (f) is precisely the density profile plotted in Fig. 11(b).

the center of the QPC between the two density maxima (shaded region in Fig. 11), and use $\lambda_{F}=2 \pi / k_{F}=4 / \bar{n}$. For Fig. 11(b), the first method yields $\lambda_{F} / 2=3.82 \bar{l}_{x}$, and the second $\lambda_{F} / 2=3.55 \bar{l}_{x}$, which are both in reasonable agreement with each other and the distance $\lambda=3.62 \bar{l}_{x}$ between the two density maxima. Thus, we conclude that the period of the density oscillations observed here can be associated with $\lambda_{F} / 2$, or equivalently wavenumber $2 k_{F}$.

In Fig. 12 we examine this behavior more systematically, using two QPCs having a comparatively long screening length of $\widetilde{l}_{s}=2.15$, but which differ slightly in $\widetilde{L}_{\text {bar }}$, i.e. in their total barrier length. For both QPCs the conductance step [Figs. 12(a),(b)] changes its shape with decreasing curvature $\widetilde{\Omega}_{x}$ and for the right QPC with smaller $\widetilde{L}_{\text {bar }}$ develops additional pronounced features in the plateau region. In Figs. 12(c)-(h) we show the corre-
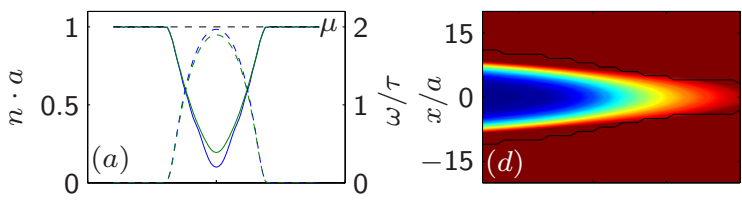

0.2
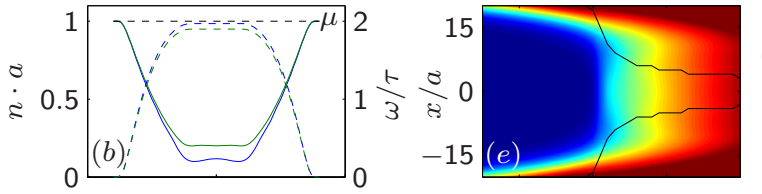

0.1
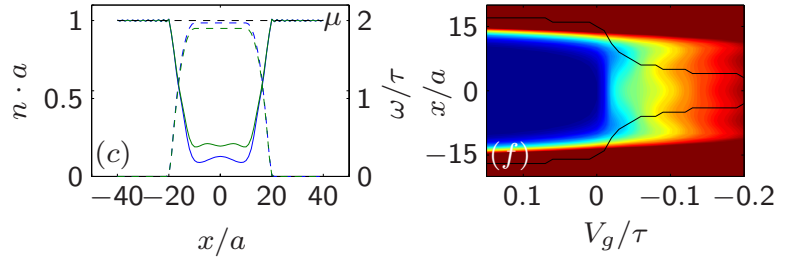

$n \cdot a$

Figure 13. (a-c) Barrier shapes (dashed lines) and corresponding noninteracting densities (solid lines) for almost open QPCs with (a) a parabolic barrier top, (b) a flat barrier top with wide flanks, (c) and a flat barrier top with steep flanks. (d-f) Density profiles corresponding to these three barrier shapes, plotted as functions of position and gate voltage. In these plots, $\lambda_{F} / 2$ is again indicated by the distance between the black lines. The flat barrier top with steep flanks of panel (c) yields pronounced Friedel oscillations in the density profile shown in panel (f), which resemble the density oscillations caused by the long-range interaction in the open regime of the QPCs of Fig. 12(e-h). This suggests that for the latter, the renormalized barriers have a rather flat tops with steep flanks.

sponding densities (color scale) as functions of gate voltage and longitudinal position, and find that with decreasing curvature $\widetilde{\Omega}_{x}$ the density develops oscillations. The period of these oscillations is again set by $\lambda_{F} / 2$, which is indicated in Figs. 12(c)-(h) by the distance between the black lines. While for the right QPC the two density maxima follow very accuratly the black lines, in the left QPC they lie slightly further apart than $\lambda_{F} / 2$. The reason for this might be that the left QPC is slightly longer $\left(\widetilde{L}_{\mathrm{bar}}\right.$ is larger $)$, giving the electrons in the center more space to form the two repelling density maxima, but not enough space to fit a third density maximum into the available region. In summary, we find that when increasing the geometric proportions of the QPC compared to the scale set by the interactions, i.e. when decreasing $\widetilde{\Omega}_{x}$, the conductance develops additional features in the plateau region, and simultaneously density oscillations arise on a scale set by $\lambda_{F} / 2$.

We interpret the $2 k_{F}$ density oscillations seen in Fig. 11(b) as Friedel oscillations generated by the inhomogeneity induced by the renormalized QPC potential. A similar interpretation was envoked in Iqbal et al. [30] where they also found a wavelength $\lambda_{F} / 2$, or equivalently a wavenumber of $2 k_{F}$, for their spin polarized, emergent localized states (ELS) obtained from SDFT calculations in long QPCs.

To support this interpretation, we show in Figs. 13(a-c) some density profiles (solid lines) obtained for a QPC model of noninteracting electrons 


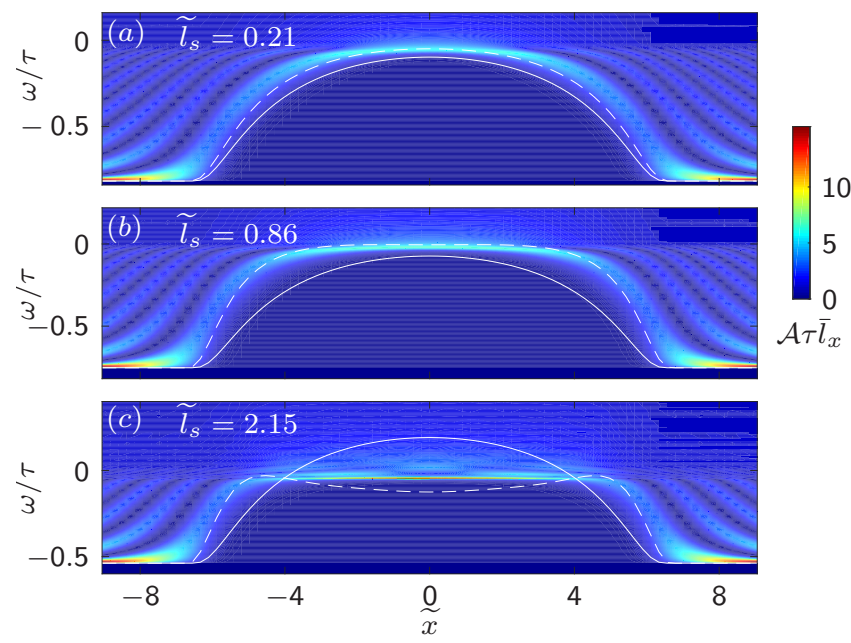

Figure 14. Interacting LDOS in the static approximation [Eq. (67)], shown as function of position and energy (color scale), for three different values of the screening length $\widetilde{l}_{s}$. Solid white lines show the bare potential $V_{j}$, and dashed white lines $V_{j}+\Sigma_{j j}$, as functions of position. The physical parameters used for this plot correspond to those of Fig. 10(b), with the gate voltage was set to $\widetilde{V}_{g}=-1.91$ in (a), $\widetilde{V}_{g}=-1.43$ in (b) and $\widetilde{V}_{g}=3.73$ in (c). (The latter two correspond to the red and blue markers in Fig. 10.) The shape of the band bottom reflects that of the renormalized barrier. (The fact that the renormalized barrier top lies below the bare barrier top in (c) is due to the artifact of static fRG discussed in section IV.B.)

traversing a QPC, comparing three different barrier shapes (dashed lines): (a) a parabolic top, (b) a flat top with a slow transition to broad flanks, and (c) a flat top with a rather quick transition to steep flanks. For a given gate voltage, the overall shape of the density profile mirrors that of the barrier top for all three cases. Moreover, pronounced additional density oscillations arise for case (c). Panels (d) to (f) show the corresponding evolution of such density profiles with gate voltage. For gate voltages where the QPC is sufficiently open that the density in the center is not very low, the density oscillations seen in Figs. 13(c) and 13(f) are reminiscent, respectively, of those seen in Figs. 11(b) and $12(\mathrm{c}-\mathrm{h})$ for QPCs with interactions whose range is longer than the characteristic QPC length (i.e. with $\tilde{l}_{s}>1$ ). This supports the interpretation offered above that such QPCs indeed have renormalized barriers with rather flat tops and steep flanks. However, for higher gate voltages where the $\mathrm{QPC}$ is beginning to close off and the density in the center becomes very low, we see a qualitative difference between the density profiles shown in Fig. 13(f) and those of Figs. 12(c-h): the former shows a weak density maximum, whereas the latter do not, because in the regime of very low densities, the Hartree-type renormalization of the barrier shape is not yet strong enough to generate a flattish barrier top.

To further explore our hypothesis concerning the occurence of a renormalized barrier with a flattened top and steep flanks, we have studied the influence of the screening length, $\widetilde{l}_{s}$, on the interacting LDOS in the static approximation,

$$
\mathcal{A}_{j}(\omega)=-\frac{1}{\pi} \operatorname{Im} G_{j j}\left(\omega+i 0^{+}\right)=-\frac{1}{\pi} \operatorname{Im}\left[\omega-h^{0}-\Sigma\right]_{j j}^{-1},
$$

where $h_{i j}^{0}=\delta_{i j} V_{j}-\tau\left[\delta_{i, j+1}+\delta_{i, j-1}\right]$ is the bare singleparticle Hamiltonian, and $\Sigma_{i j}$ is the static self-energy at the end of the RG flow [31]. Fig. 14 shows the LDOS (color scale) as function of position and energy, for three values of the screening length, $\widetilde{l}_{s}$. We interpret the shape of the effective band bottom as indicative of the shape of the effective barrier. We observe that with increasing $\widetilde{l}_{s}$, the effective barrier top indeed does become strikingly flat over an extended region of space centered on the middle of the QPC, ending in rather steep flanks, as anticipated above. For comparison, solid white lines show the bare potential $V_{j}$ with its parabolic top. Moreover, dashed white lines show $V_{j}+\Sigma_{j j}$, to illustrate the contribution of the diagonal elements of the self-energy to the renormalization of the potentatial barrier. However, while $V_{j}+\Sigma_{j j}$ does show a trend toward barrier flattening with increasing screening length, for the largest $\widetilde{l}_{s}$ value [Fig. 14(c)] it leads to a shallow local minimum at $\widetilde{x}=0$, reminiscent of a $\mathrm{QD}$-like barrier shape. To correctly capture the shape of the band bottom, which shows no such local minimum, the off-diagonal elements of the self-energy have to be taken into account, too. This is done when computing the LDOS according to Eq. (67), which involves inverting the entire matrix $\omega-h^{0}-\Sigma$ before taking diagonal elements.

The above results show that long-range interactions can have a rather striking flattening effect on the effective barrier shape, and that long flat barriers lead to interesting density oscillations. It would thus be interesting to study the geometric crossover from a QPC to a homogeneous wire obtained by making the QPC length $\bar{l}_{x}$ very long, or by using flat-topped bare barriers of increasing width. In a paper by Schulz [32], concerning Wigner crystal physics in 1D, it was predicted that in a homogeneous 1D model with long-ranged Coulomb interactions in the low-density limit, the density-density correlator $\langle\rho(x) \rho(0)\rangle$ contains both $2 k_{F}$ and $4 k_{F}$ oscillations. The latter decay more slowly with $x$, and are argued by Schulz to lead to a Wigner crystal in a homogeneous system. During the aforementioned geometric crossover from a QPC to a long wire, well-developed $4 k_{F}$ density oscillations can be expected to emerge, which could be regarded as precursors for the formation of a Wigner crystal. A systematic study of this behavior would be extremely interesting, but falls beyond the scope of this paper and is left for future study. In particular, future work would have to incorporate screening also due to higher transport channels, leading to a shorter-ranged interaction, so that the effects discussed above would likely turn out to be somewhat less pronounced than found here.

\section{CONCLUSION AND OUTLOOK}

Building on previous works [7, 8], we have introduced an improved approximation scheme for 3rd-order trun- 
cated fRG. We use an extended coupled ladder approximation (eCLA), splitting the fRG-flow into three channels depending on the internal index structure. When treated independently, each of these channels behaves as in the random phase approximation. The complexity of the eCLA scheme depends on the amount of feedback admitted between the individual channels. For the frequency dependence, we only used static feedback between the channels. In order to control the amount of feedback in the spatial structure, we have introduced the feedback length $L$. In the case $L=0$ we get the minimal feedback between the channels, corresponding to the CLA of previous works [8], whereas for $L \rightarrow N-1$ we recover the full spatial vertex flow in 2 nd order.

For actual computations, we restricted ourselves to static fRG, i.e. in addition to using only a static feedback between the channels we also neglected the frequency dependence of the vertices altogether. In this additional approximation, we calculated the zero-temperature Green's function at the chemical potential, which is the relevant quantity in order to compute the linear conductance of the system.

We first applied our new method to a QPC model with onsite interactions, which has extensively been studied in the past. Here, we observed that the longer-ranged feedback leads to a quantitative but not qualitative change as long as both methods are convergent for the respective parameters. In particular, we observed for barriers with characteristic lengths between $4-10$ sites that convergence in $L$ is achieved onces $L$ becomes comparable to $l_{x}$. Additionally, we observed that the enhanced feedback stabilizes the fRG-flow and therefore leads also to convergence in parameter regimes which could not be studied with the $L=0$ method. To illustrate this increased stability, we studied QPC-QD crossovers analogous to those discussed by Heyder et al. in Ref. [19] using the CLA. There, the convergence of the fRG flow suffers especially from the high LDOS at the chemical potential that occurs during the crossover when the barrier top becomes flat in an extended region close to the chemical potential. Our stabilized flow, however, enabled us to study this type of transition. In particular, we succeeded to study regimes of very shallow dots, containing only a few electrons, and observed the Kondo plateau in the conductance expected for such dots.

Finally, in order to test the full potential of our improved feedback, we applied it to a QPC with finiteranged interactions. The most striking observation was that for a relatively flat QPC in the regime of low density and sufficiently long-ranged interactions, the conduc- tance reaches a preplateau somewhat below $g=1$ (before slowly climbing towards $g=1$ ), accompanied by the onset of oscillations in the density. The wavelength of these density oscillations was determined to be approximately $\lambda_{F} / 2$, admitting an interpretation as Friedel oscillations arising from a renormalized barrier shape with a rather flat top and steep flanks. This behavior is consistent with that observed by Iqbal et al. [30] in SDFT calculations for their emergent localized states (ELS) in a spin-polarized QPC. It would be of great interest to explore these type of effects more systematically in the future, within a more realistic model that incorporates the effects of higher transport modes when deriving the effective screened interaction for the lowest-lying transport mode. In particular, the geometric crossover between a QPC potential and a homogeneous quantum wire, expected to show Wigner crystallization, could be explored in this fashion. However, it remains to be seen whether fRG will be able to cope with the truly homogeneous limit; such a study will presumably also have to employ tools more powerful than fRG, such as the density matrix renormalization group.

By way of an outlook to future technical fRG developments, let us remark that it would be desirable to find ways of avoiding an fRG artifact that is present in our results: upon turning on a long-ranged interaction, the position of the conductance step shifts not to smaller gate voltages, as physically expected, but to larger ones. We suspect that this is artefact results from our use of static fRG. A next possible step to remedy this problem could be to change from static to dynamic fRG, i.e. to implement the frequency dependence of the vertices. Moreover, it would also be possible to use our enhanced feedback scheme in the context of Keldysh fRG, which is additionally able to treat the temperature dependence and non-equilibrium behavior of QPCs. This would be numerically challenging since the Keldysh scheme in the $L=0$ implementation is already very costly by itself. However, one might profit from the fact that the most expensive part of the Keldysh calculation scales with $\mathcal{O}\left(L^{2}\right)$, and not with $\mathcal{O}\left(L^{3}\right)$ as in our case. Work in that direction is currently in progress.

\section{ACKNOWLEDGMENTS}

We thank Jan Heyder, Volker Meden, Yigal Meir and Dennis Schimmel for very helpful discussions. We acknowledge support from the DFG via SFB-631, SFB-TR12, De730/4-3, and the Cluster of Excellence Nanosystems Initiative Munich.
[1] W. Metzner, M. Salmhofer, C. Honerkamp, V. Meden, and K. Schönhammer, Rev. Mod. Phys. 84, 299 (2012).

[2] S. Andergassen, T. Enss, C. Karrasch, and V. Meden, in Lecture Notes in Physics, Berlin Springer Verlag, Lecture Notes in Physics, Berlin Springer Verlag, Vol. 645, edited by B. Barbara, Y. Imry, G. Sawatzky, and P. C. E.
Stamp (2008) p. 1, cond-mat/0612229.

[3] C. Karrasch, T. Enss, and V. Meden, Physical Review B (Condensed Matter and Materials Physics) 73, 235337 (2006).

[4] S. G. Jakobs, M. Pletyukhov, and H. Schoeller, Phys. Rev. B 81, 195109 (2010). 
[5] C. Karrasch, Transport Through Correlated Quantum Dots - A Functional Renormalization Group Approach, Master's thesis, Georg-August Universität Göttingen (2006), arXiv:cond-mat/0612329v1.

[6] C. Karrasch, The Functional Renormalization Group for Zero-Dimensional Quantum Systems in and out of Equilibrium, Ph.D. thesis, RWTH Aachen (2010).

[7] F. Bauer, J. Heyder, E. Schubert, D. Borowsky, D. Taubert, B. Bruognolo, D. Schuh, W. Wegscheider, J. von Delft, and S. Ludwig, Nature 501, 73 (2013).

[8] F. Bauer, J. Heyder, and J. von Delft, Phys. Rev. B 89, 045128 (2014).

[9] C. Karrasch, R. Hedden, R. Peters, T. Pruschke, K. Schnhammer, and V. Meden, Journal of Physics: Condensed Matter 20, 345205 (2008).

[10] Y. Meir, Journal of Physics: Condensed Matter 20, 164208 (2008).

[11] C. Sloggett, A. I. Milstein, and O. P. Sushkov, The European Physical Journal B 61, 427 (2008).

[12] K. Aryanpour and J. E. Han, Phys. Rev. Lett. 102, 056805 (2009).

[13] B. J. van Wees, H. van Houten, C. W. J. Beenakker, J. G. Williamson, L. P. Kouwenhoven, D. van der Marel, and C. T. Foxon, Phys. Rev. Lett. 60, 848 (1988).

[14] D. A. Wharam, T. J. Thornton, R. Newbury, M. Pepper, H. Ahmed, J. E. F. Frost, D. G. Hasko, D. C. Peacock, D. A. Ritchie, and G. A. C. Jones, Journal of Physics C: Solid State Physics 21, L209 (1988).

[15] M. Büttiker, Phys. Rev. B 41, 7906 (1990).

[16] K. J. Thomas, J. T. Nicholls, M. Y. Simmons, M. Pepper, D. R. Mace, and D. A. Ritchie, Phys. Rev. Lett. 77, 135 (1996).

[17] N. J. Appleyard, J. T. Nicholls, M. Pepper, W. R. Tribe, M. Y. Simmons, and D. A. Ritchie, Phys. Rev. B 62, R16275 (2000).

[18] S. M. Cronenwett, H. J. Lynch, D. Goldhaber-Gordon, L. P. Kouwenhoven, C. M. Marcus, K. Hirose, N. S.
Wingreen, and V. Umansky, Phys. Rev. Lett. 88, 226805 (2002).

[19] J. Heyder, F. Bauer, E. Schubert, D. Borowsky, D. Schuh, W. Wegscheider, J. von Delft, and S. Ludwig, Phys. Rev. B 92, 195401 (2015), arXiv:1409.3415 [cond-mat.str-el].

[20] F. Bauer, Microscopic Origin of the 0.7 Anomaly in Quantum Point Contacts, Ph.D. thesis, LMU-München (2014).

[21] S. G. Jakobs, V. Meden, and H. Schoeller, Phys. Rev. Lett. 99, 150603 (2007).

[22] C. Husemann and M. Salmhofer, Phys. Rev. B 79, 195125 (2009).

[23] T. R. Morris, International Journal of Modern Physics A 09, 2411 (1994).

[24] J. Dormand and P. Prince, Journal of Computational and Applied Mathematics 6, 19 (1980).

[25] J. Kondo, Prog. Theor. Phys. 32, 37 (1964).

[26] S. Datta, Electronic Transport in Mesoscopic Systems, Cambridge Studies in Semiconductor Physics and Microelectronic Engineering (Cambridge University Press, 1997).

[27] O. Goulko, F. Bauer, J. Heyder, and J. von Delft, Phys. Rev. Lett. 113, 266402 (2014), arXiv:1408.0746v1 [condmat.mes-hall].

[28] A. M. Lunde, A. D. Martino, A. Schulz, R. Egger, and K. Flensberg, New Journal of Physics 11, 023031 (2009).

[29] K. Hirose, Y. Meir, and N. S. Wingreen, Phys. Rev. Lett. 90, 026804 (2003).

[30] M. J. Iqbal, R. Levy, E. J. Koop, J. B. Dekker, J. P. de Jong, J. H. M. van der Velde, D. Reuter, A. D. Wieck, R. Aguado, Y. Meir, and C. H. van der Wal, Nature 501, 79 (2013).

[31] As a technical caveat, we note that static fRG is expected to be less reliable than dynamic fRG for quantities computed at $\omega \neq 0$, hence it would be highly desirable to repeat this calculation using Keldysh fRG. Work along these lines is currenty in progress.

[32] H. J. Schulz, Phys. Rev. Lett. 71, 1864 (1993). 